\title{
Campos Conceituais e Indução Eletromagnética: Classificação de Problemas em Eletrodinâmica
}

\author{
Conceptual Fields and Electromagnetic Induction: Classification of Problems \\ in Electrodynamics
}

Palavras-chave Resumo Esta pesquisa objetiva apresentar a construção de um Campos referencial teórico-metodológico para classificar problemas de Conceituais; eletrodinâmica dirigidos para o Ensino Médio e para o Ensino Indução Superior. A abordagem se baseia na teoria dos campos conceituais Eletromagnética; e inclui a classificação de tarefas em termos das operações de Situações-Problema pensamento essenciais à sua resolução e dos parâmetros destas. Neste contexto, a relevância dessa classificação à luz da teoria citada está em levar em conta o aspecto referencial dos conceitos no processo de ensino-aprendizagem, ou seja, considerar o papel das situações na tomada de sentido ocorrente nas aprendizagens conceitual e procedimental. Propomos quatro classes primárias de situações, quais sejam, descrição de interações eletromagnéticas, representação analógica de campos eletromagnéticos, representação simbólica de campos eletromagnéticos e cálculo de campos eletromagnéticos. Estas classes são irredutíveis umas às outras, mas podem surgir concomitantemente em uma mesma situação. Cada classe primária é subdividida em classes secundárias de tarefas com base nos parâmetros que podem ocorrer para elas e as últimas são ordenadas por complexidade epistemológica. A construção de um campo conceitual para a indução eletromagnética implica para a área de Ensino de Ciências em três possibilidades imediatas, nomeadamente, o estudo do conhecimento operatório vinculado a este conceito, a classificação psicológica de situações e a abordagem de processos de oportunismo da conceitualização. 
Keywords Abstract This research aims to present a theoretical-methodological Conceptual Fields; framework for classifying situations in electrodynamics. The Electromagnetic approach is based on the theory of conceptual fields and includes the

Inductions; classification of tasks in terms of the thought operations necessary for

Problem-Situations. its resolution and its parameters. In this context, the relevance of this classification in the light of the mentioned theory is in regarding the referential feature of the concepts in teaching-learning processes, that is, considering the role of situations in sense making of conceptual and procedural learning. We propose for primary classes of situations, namely, description of electromagnetic interactions, analogic representation of electromagnetic fields, symbolic representation of electromagnetic fields and calculation of electromagnetic fields. These classes are irreducible among them but can occur simultaneously in the same situation. Each primary class is subdivided in secondary classes of tasks based on the parameters that can be attached to them and the latter are ordered by epistemological complexness. Building a conceptual field for electromagnetic induction implies in three immediate possibilities for Science Teaching research, namely, the study of the operatory knowledge attached to this concept, the psychological classification of situations and the addressing of processes of opportunism of conceptualization.

\section{Introdução}

A teoria eletromagnética (TE) é um campo do conhecimento importantíssimo para a compreensão do mundo contemporâneo e do fazer científico-tecnológico, além de estar nos mais diversos currículos escolares e universitários (em cursos de ciências exatas) ao redor do mundo. No que tange às pesquisas sobre o ensino e a aprendizagem do conceito de indução eletromagnética (IE), central na TE, sabe-se que a despeito de haver um número incipiente de investigações relativas à IE, tais estudos identificam tanto a existência de concepções alternativas específicas nas estruturas cognitivas de estudantes de diversos níveis educacionais, quanto dificuldades gerais na aprendizagem do conceito de campo eletromagnético (Zuza et al., 2012a).

Dentre os obstáculos gerais de aprendizagem apontados por estas pesquisas, são mais recorrentes a incapacidade de relacionar conceitos centrais da teoria eletromagnética (Jelicic et al., 2017; Zuza et al., 2012a), a inabilidade em diferenciar epistemologicamente e ontologicamente os programas de pesquisa coulombiano e Maxwelliano (Jelicic et al., 2017; Zuza et al., 2012a), a inaptidão em reconhecer fenômenos de indução eletromagnética (Zuza et al., 2014; Zuza et al. 2012a) e a dificuldade em distinguir entre níveis empírico e interpretativo (Zuza et al, 2014). Destacamos que o entendimento mais elaborado da origem e da evolução desses entraves na aprendizagem pode dar pistas para a realização de processos de ensino mais eficazes (Vergnaud, 2009; Vergnaud, 1982). 
Embora várias investigações sustentem que tanto a aprendizagem quanto o desenvolvimento cognitivo se processam a partir de situações (Vergnaud, 2013), poucos estudos têm considerado esta questão no contexto do eletromagnetismo (Pantoja \& Moreira, 2019). A maioria dos estudos em ensino e aprendizagem de IE se foca em descrever o conteúdo das relações conceituais (significado) e as representações delas decorrentes (significante) sem considerar o aspecto referencial do conceito, ou seja, sua referência à realidade.

Diversos estudos com muita sanidade teórico-metodológica ${ }^{1}$ têm abordado a questão da mesma forma, ou seja, sem levar em conta os referentes do conceito. Não queremos deixar a impressão de que tais trabalhos não sejam importantes ou que seus resultados não tenham validade, mas apontar que estes achados podem ser mais bem interpretados se levarmos em conta o caráter situacional dos conceitos, isto é, se consideramos que conceitos se referem a situações e que estas influenciam fortemente na forma com que são aprendidos e ensinados (Vergnaud, 1982; Vergnaud, 2009).

Uma forma frutífera de contornar esta questão é elaborando um campo conceitual para a IE (Vergnaud, 2013). Esta perspectiva admite que podemos entende-la como dividida em campos conceituais constituídos pelas situações (referentes), significados e representações (significantes) vinculados a este conceito (Vergnaud, 2009). As situações compõem o aspecto referencial da IE, enquanto os significados e significantes compõem seu aspecto representacional (Vergnaud, 2013). Com base nesta problemática, propomos a seguinte questão de pesquisa: "que tipos de situações, invariantes operatórios e representações compõem o campo conceitual da IE?”. Assim, este trabalho tem como objetivo a estruturação das situações de IE em classes de problemas. A seguir, apresentamos a revisão de literatura, seção na qual discutimos como os trabalhos em ensino de eletromagnetismo têm sido comumente desenvolvidos e os resultados mais recorrentes dessas pesquisas.

\section{Revisão da Literatura}

Foi feita uma revisão de literatura na qual foram cruzados dados obtidos no Education Resources Information Center (ERIC) com o sistema de qualificação da Coordenação de Aperfeiçoamento de Pessoal de Nível Superior (CAPES), o qualis. Os artigos envolvidos na revisão são oriundos de revistas qualificadas como A1 e A2 (estratos mais altos) e foram encontrados na plataforma ERIC após ativação do filtro para seleção dos trabalhos publicados nos últimos dez anos.

Dentre esses estudos, encontramos uma revisão de literatura feita dos anos 90 até 2012, devida a Zuza et al. (2012a), e outras pesquisas relacionadas à temática da indução eletromagnética. Desses artigos, selecionamos somente aqueles associados às concepções de estudantes. Logo, investigações sobre resultados de propostas didáticas diferenciadas, propostas didáticas não testadas efetivamente, análise históricoepistemológica de assuntos de eletromagnetismo e análise curricular (Pantoja et al.,

1 Para revisões recentes, verificar Jelicic et al. (2017) e Zuza et al. (2012a). 
2011) foram descartados. Assim se fez, pois o escopo deste trabalho compreende esta categoria selecionada para análise.

Diversas dificuldades enfrentadas por estudantes na aprendizagem do conceito de indução eletromagnética e de ideias correlatas foram encontradas nessas pesquisas. Esses obstáculos são colocados em termos de proposições consideradas verdadeiras pelos alunos e estas relacionam categorias consideradas pertinentes por eles. O primeiro que destacamos é a relação entre a lei de Faraday e a área à qual se refere o cálculo da FEM induzida. Muitos alunos universitários consideram que a área sobre a qual computamos o fluxo equivale à área do circuito quando, na verdade, a superfície a considerar-se deve ser igual à área varrida pelo movimento do circuito (Galili et al., 2006; Saarelainen et al., 2007; Zuza et al., 2012a).

Outros entraves na aprendizagem são derivados de incompreensão das relações entre campos eletromagnéticos e forças eletromagnéticas. Existe uma alta frequência de respostas indicando que diversos estudantes, dos mais diversos níveis, consideram que os conceitos de campo e de força são similares e, portanto, um deles deve ser supérfluo (Guisasola et al., 2011; Zuza et al., 2012a), e campos eletrostáticos e não-eletrostáticos como indistinguíveis (Jelicic et al., 2017; Thong \& Gunstone, 2008; Zuza et al., 2012a). Outras dificuldades perpassam pela consideração dos alunos de que força elétrica e força magnética não podem ser diferenciadas (Bagno \& Eylon, 2000; Jelicic et al., 2017; Zuza et al., 2012a;). Aspectos substancialistas também têm sido identificados, tais como os na assunção de que $a$ força eletromagnética é realizada por meio de contato com o campo (Bagno \& Eylon, 2000; Jelicic et al., 2017; Loftus, 1996; Thong \& Gunstone, 2008; Zuza et al., 2012a).

Existe uma série de dificuldades explicitadas pelos estudantes com respeito à lei de Faraday, tais como as expressas pelas ideias de que: campo magnético produz corrente elétrica induzida (Mauk \& Hingley, 2005; Zuza, Guisasola, Michelini \& Santi, 2012b); fluxo magnético e campo magnético são indistinguíveis (Albe, Venturini \& Lascours, 2001; Jelicic et al., 2017; Loftus, 1996; Thong \& Gunstone, 2008; Venturini e Albe, 2002; Zuza et al, 2014); o fluxo magnético é um conceito meramente matemático (Albe et al., 2001; Venturini \& Albe, 2002; Zuza et al., 2012b); a variação temporal do fluxo magnético equivale ao próprio fluxo magnético (Saarelainen et al., 2007; Zuza et al., 2012b); o fluxo magnético é entendido como fluidez do campo magnético (Albe et al., 2001; Loftus, 1996; Thong \& Gunstone, 2008; Venturini \& Albe, 2002; Zuza et al, 2014); o sentido da corrente elétrica se opõe à variação do fluxo magnético (Mauk \& Hingley, 2005; Zuza et al., 2012b). Dessa forma, os impedimentos dos estudantes aparecem nas três facetas do eletromagnetismo, quais sejam, compreensão de fontes de campo eletromagnético, descrição das forças eletromagnéticas e trocas de energia eletromagnética (Nousianen \& Koponen, 2017).

Trabalhos como os desenvolvidos por Guisasola et al. (2011) são representativos da tendência das pesquisas realizadas em concepções de estudantes sobre a indução eletromagnética e, por isso, citamos esse trabalho como exemplo. Nessa investigação, são 
identificadas dificuldades em determinar a natureza microscópica da força eletromotriz induzida em situações IE, o que não acontece quando o enunciado solicita a natureza macroscópica da FEM.

Para os autores da pesquisa, a explicação macroscópica da IE é feita em termos da variação do fluxo magnético, a qual leva a uma FEM, enquanto a microscópica é feita em termos da ação da força de Lorentz responsável pelo estabelecimento da aceleração dos elétrons do circuito (Guisasola et al., 2011). Os pesquisadores evidenciaram de maneira metodologicamente bem ajustada que, mesmo tendo sido apresentados às duas explicações, estudantes do ensino superior conseguiam compreender claramente a abordagem macroscópica (entre 64\% e 66\% dos estudantes), mas não tinham êxito em desenhar explicações com conteúdo microscópico (entre 4\% e 13\% dos alunos), frente à situação proposta a seguir:

quando se muda a direção de uma espira circular numa região de campo magnético, encontra-se experimentalmente que o amperímetro $G$, ligado à espira, registra uma corrente elétrica. Explique de onde vêm as forças que movem as cargas elétricas na espira² (Guisasola et al., 2011).

Figura 1. Espira condutora girando em região de campo magnético (imagem de Guisasola et al., 2011)

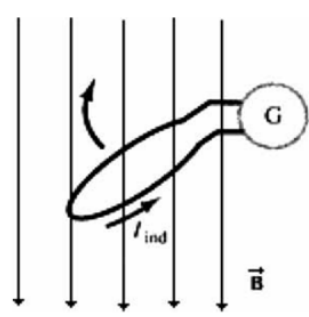

Conforme apontado pelos autores, a força de Lorentz é central na explicação microscópica, enquanto a lei de Faraday na forma integral é mais importante na explicação macroscópica. Disso, esses pesquisadores concluem que os estudantes entendem bem a lei de Faraday e não compreendem a lei de Lorentz (Guisasola et al., 2011). Isso está claro, mas a questão que fica a responder: sob que condições esse evento acontece? Se se leva em conta que um campo conceitual é um conjunto de situações, invariantes e representações, a mudança ligeira da situação acarreta modificar os invariantes e representações usados para resolvê-la (Vergnaud, 2009), como parece ser o caso da questão.

Um contraexemplo a essa tendência de pesquisa é a investigação encabeçada por Scaife e Heckler (2010). Estes autores detectaram por meio de experimentos pedagógicos que em uma tarefa na qual se deva determinar a força magnética exercida por um campo magnético sobre uma carga elétrica em movimento, o simples fato de propor o problema com a fonte explícita e as linhas de campo implícitas é suficiente para deixa-lo mais

2 Tradução livre feita por conta do autor. 
difícil em relação à situação na qual a fonte está implícita e as linhas de campo explícitas (Scaife \& Heckler, 2010).

Em cinco experimentos pedagógicos realizados, Scaife e Heckler (2010) apresentaram duas representações distintas do problema da interação entre um campo magnético e uma carga elétrica em movimento para dois grupos diferentes. Um dos grupos recebia o problema de uma carga elétrica movendo-se ao longo do eixo $x$ em uma região de campo magnético na representação do tipo linhas de campo (Figura 2 - esquerda), enquanto o outro tinha acesso à representação do tipo fonte (Figura 2 direita).

Para avaliar a performance dos alunos, os pesquisadores usaram entrevistas e respostas escritas. Os resultados apontam resultados baixos na performance de ambos os grupos, mas uma diferença significativa a favor do grupo com a representação de linhas de campo em quatro instantes diferentes ${ }^{3}$ (Scaife \& Heckler, 2010), ou seja, o mesmo problema colocado de duas formas distintas parece mobilizar distintos conhecimentos prévios, quais sejam, o de que os pólos dos ímãs são cargas elétricas e de que as cargas são "puxadas" ao longo das linhas de campo. Nesse caso, as primeiras considerações parecem ser mais efetivamente acessadas e mais dificilmente obliteradas (Scaife \& Heckler, 2010).

Figura 2. Portador pontual de carga elétrica movendo-se para a esquerda com velocidade constante em região de campo magnético representada de duas formas: diretamente, por linhas de campo (esquerda); e indiretamente, por explicitação da fonte (direita) (imagem adaptada de Scaife e Heckler, 2010)
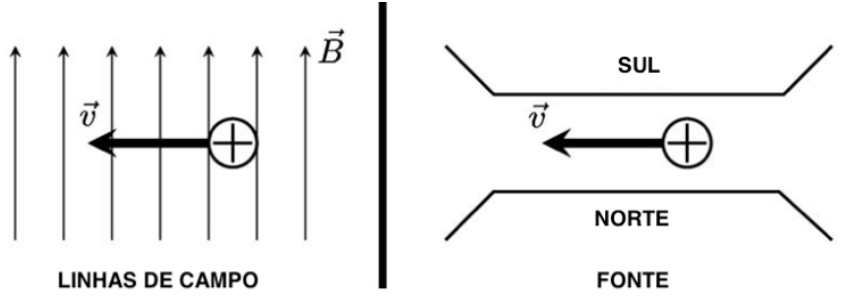

Este tipo de trabalho é muito importante, no entanto, por não existir qualquer proposta de classificação de situações em IE, análises desse tipo ainda carecem de maior estruturação dos parâmetros característicos de situações da $\mathrm{TE}^{4}$. Como essa problemática poderia ser mais bem entendida? Se tivermos uma estrutura conceitual didática de referência para a IE, um campo conceitual, o que até hoje ainda não existe na literatura.

Entendo que estes estudos são providos de alta consistência teórico-metodológica e apresentam valiosos resultados sobre as concepções sustentadas pelos alunos, no entanto, eles desconsideram o aspecto referencial dos conceitos e falham em discutir como estes significados e significantes são trabalhados em situação, conforme já

3 A saber, antes da instrução (I), após a instrução sobre campo elétrico e antes da instrução sobre campo magnético (II), logo após a instrução sobre campo magnético (III), dois meses depois da instrução campo magnético.

4 Em TE, consideramos a eletrodinâmica como o estudo dos campos não eletrostáticos, o que inclui campos elétricos e magnéticos variáveis com o tempo, além de situações de FEM de movimento (Resnick et al., 2006). 
apontado. Portanto, é necessário estabelecer um modelo de análise das situações para que estudemos como as estruturas de conhecimento que os estudantes possuem se adaptam a essas situações, pois assim estaremos aptos a reinterpretar estes resultados num quadro de referência mais adequado. Este modelo se baseia na teoria dos campos conceituais, o referencial teórico deste trabalho.

\section{Referencial teórico}

A teoria dos campos conceituais (TCC) se ocupa de descrever processos de conceitualização, o estabelecimento de referência ao real através da representação. Nesta perspectiva, a conceitualização se torna o ponto fundamental da cognição, pois é o âmago do desenvolvimento cognitivo. Esta teoria parte da premissa de que as operações de pensamento independentes de conteúdo postuladas por Piaget pouco facilitam a tarefa de compreender dificuldades específicas dos estudantes (Vergnaud, 2009).

A TCC admite que os conceitos são elementos que estabelecem a conexão entre o real e a representação. Desta forma, este aspecto de referência reside nas situações que os tornam úteis e significativos. Portanto, a noção de interação entre realidade e representação deve ser reestruturada na noção de conceito. Assim, o último passa a ser entendido como uma terna de conjuntos de referentes, de significados e de significantes (Vergnaud, 2013).

É possível expressar o conceito $C$ por $C(S, I, R)$, onde $S$ é seu referente, as situações que o tornam útil e significativo; I é o seu significado, o conjunto de invariantes operatórios sob o qual subjazem sua operacionalidade e conteúdo semântico; e $R$ é o seu significante, o conjunto de representações linguísticas, simbólicas ou analógicas que representam $I$ e de $S$ (Vergnaud, 2009). Portanto, $S$ é a realidade, $I$ e $R$ são a representação.

O campo conceitual pode ser entendido como uma delimitação epistemológica e psicológica dos conceitos. Assim, é definido como um conjunto de situações, invariantes operatórios, representações, operações de pensamento e as complexas relações entre esses elementos (Vergnaud, 2013). A parte epistemológica do campo conceitual pode ser compreendida como aquela volta à descrição dos elementos de conhecimentos comuns a cada classe de situações. A parte psicológica inclui a gradação das dificuldades dos estudantes em lidar com cada uma das classes. Fazer a descrição desta maneira é uma tarefa árdua e requer a cooperação de diversos pesquisadores (Vergnaud, 1982), pois há várias formas de analisar dificuldade e de descrever situações. Por esta razão, nossa proposta de classificação é somente um primeiro passo neste empreendimento.

O conhecimento científico pode ser pensado em termos de campos conceituais, pois há classes de situações relacionando distintos significados e significantes. Ademais, estas podem ser estudadas independentemente, a despeito de correlações existentes entre campos conceituais. Na biologia, por exemplo, a reprodução de vegetais pouco ou nada tem a ver com a reprodução animal. Na Física, com exceção do famoso efeito Joule, o estudo dos conceitos de corrente elétrica e de temperatura podem ser estudados praticamente de maneira separada ${ }^{5}$. Estes recortes são artificiais, mas temos que estar

5 Historicamente estes campos conceituais foram estudados praticamente de forma separada até meados do século 
conscientes que é impossível estudar tudo a uma só vez, portanto, é importante ter certa dose de pragmatismo para abordar estas questões, já que elas têm utilidade em problemas pedagógicos reais (Vergnaud, 2009).

As dificuldades de aprendizagem são bastante diferentes entre campos conceituais, pois estes diferem epistemologicamente e ontologicamente uns dos outros. Isto torna impossível subsumir os processos cognitivos a aspectos gerais lógicos, logo, o conteúdo específico a ser aprendido assume importância central na análise. A base conceitual implícita ou explícita sobre a qual repousa a conceitualização está nos invariantes operatórios, classificados em teoremas-em-ação e em conceitos-em-ação. Os primeiros são proposições consideradas verdadeiras sobre o real, enquanto os últimos são categorias tomadas como pertinentes em relação ao real. Um não se reduz ao outro, pois as categorias possuem status de pertinência ou impertinência, enquanto as proposições possuem status de verdade ou falsidade (Vergnaud, 2009).

A atividade humana incorpora tanto elementos explícitos e verbalizados quanto implícitos e enativos ${ }^{6}$. A forma predicativa do conhecimento está associada aos dois primeiros e a forma operatória da cognição está relacionada aos dois últimos. A conceitualização envolve ambas as formas de cognição, desta feita, a TCC se apropria do conceito de esquema para descrevê-la. A partir dos quatro enunciados complementares a seguir, o esquema pode ser definido como: 1) uma totalidade dinâmica funcional; 2) a organização invariante da ação para uma determinada classe de situações; 3) composto de metas, de regras de ação do tipo se... então, de invariantes operatórios e de possibilidades de inferência, e estes são seus aspectos intencional, gerativo, epistemológico e computacional, respectivamente; 4) uma função que tem seus valores de entrada em um espaço temporalizado de n dimensões e seus valores de saída em um espaço temporalizado de n' dimensões ( $n$ e $n$ ' são grandes).

As noções de cálculo numérico e de cálculo relacional são essenciais para a construção de um campo conceitual. O primeiro é definido como a computação de operações ordinárias para resolver uma situação, enquanto o segundo é entendido como o conjunto de operações de pensamento necessárias para manejar as relações envolvidas na resolução daquela (Vergnaud, 1982). Exemplificamos a diferença entre os dois a partir do exemplo a seguir: "João recebeu 4 reais de sua mãe. Agora ele tem 7 reais. Quanto ele tinha antes?"

Figura 3. Exemplo da diferença entre cálculo numérico, cálculo operacional e representação (adaptado de Vergnaud, 1982)

\begin{tabular}{|l|l|l|}
\hline Situação: João recebeu 4 reais de sua mãe. Agora ele tem 7 reais? Quanto ele tinha antes? \\
\hline Cálculo numérico & Cálculo relacional & Representação \\
\hline Subtração $(7-4)$ & $\begin{array}{l}\text { Achar a inversa de uma transformação } \\
\text { positiva e aplicar ao estado final. }\end{array}$ & $\square \stackrel{+4}{\longrightarrow}$\begin{tabular}{|l|}
\hline \\
\hline
\end{tabular}
\end{tabular}

XIX (Whittaker, 1910).

6 Em um sentido de ação guiada por percepção, eficaz, localizada no aqui e no agora, implícita e pouco verbalizada. 
O cálculo numérico necessário para resolver o problema é uma subtração (74). Por outro lado, o cálculo relacional envolve operações de pensamento tais como caracterizar a situação como uma transformação ligando duas medidas, identificar o estado final (7), identificar a transformação $(+4)$, inverter a transformação $(-4)$, aplicar a transformação inversa ao estado final (7+[-4]) e obter o estado inicial (3). A representação é colocada na forma de diagrama na Figura 3, mas pode ser feita de outras maneiras (Vergnaud, 1982). Passemos à descrição do modelo teórico para análise de situações.

\section{Modelo teórico para análise de situações}

A análise epistemológica das situações perpassa pela caracterização da forma pela qual os conteúdos estão estruturados. Isto envolve apontar as operações de pensamento necessárias para resolver determinada classe de situações, os significados e representações vinculados às situações, os cálculos numéricos associados às grandes classes de situações, bem como os cálculos relacionais. Esta análise dá um panorama geral sobre a complexidade das classes do campo conceitual, mas não o elucida completamente, pois uma análise psicológica também é necessária para entender as dificuldades dos estudantes. Limitamo-nos ao recorte epistemológico da análise nesta investigação, já que a abordagem simultânea destes dois aspectos seria extensa demais para um só trabalho.

As classes de situações do campo conceitual da IE foram caracterizadas com base nas operações de pensamento necessárias para resolvê-las. Distinguimos dois tipos de classes de situações, as primárias $\left(C_{p}\right)$ e as secundárias $\left(C_{S}\right)$. As primeiras compartilham as mesmas operações de pensamento, enquanto as segundas compartilham parâmetros similares além das mesmas operações de pensamento.

Dividimos as operações de pensamento em gerais $\left(h_{G}\right)$ e específicas $\left(h_{S}\right)$. As gerais identificam formas de pensamento mais amplas que distinguem entre as classes primárias de situações. As específicas envolvem os cálculos relacionais que caracterizam as operações de pensamento gerais, são mais particulares que elas e se colocam dentro da mesma classe primária de situações. Ambas estão conectadas e são expressas em termos de invariantes operatórios e representações, ou seja, dependem do conteúdo específico da eletrodinâmica (Pantoja \& Moreira, 2019).

A conceitualização depende dos parâmetros das situações, porém tal discussão não se encontra muito elaborada na TCC. Tudo o que se sabe está limitado a indicar variações no processo que surgem a partir da mudança de dados ou da estrutura da situação (Vergnaud, 1982). Propomos três tipos de parâmetros para aclarar esta ideia e prosseguir com a classificação secundária de situações. Tais parâmetros são os objetos, as variáveis e as incógnitas. A classificação secundária de situações ocorre dentro das classes primárias e se baseia nestes elementos.

Os objetos são elementos que guardam correspondência indireta com objetos materiais e reais ou supostos como tais (Bunge, 2011). São elementos idealizados que nos ajudam na aproximação com a realidade. Circuitos elétricos, fios de corrente e dipolos 
oscilantes podem ser tomados como exemplos de objetos. As variáveis são informações dadas ou passíveis de inferência sobre os objetos e necessárias para a solução de uma tarefa. O raio de uma espira de corrente e uma função descrevendo o campo magnético no tempo são exemplos de variáveis. As incógnitas são elementos desconhecidos a serem determinados pelo indivíduo por meio da manipulação de variáveis. Elas constituem respostas a perguntas ou a solicitação para estabelecimento de relações entre variáveis. As incógnitas são o que caracterizam uma situação como tal.

As incógnitas vão depender do que seja solicitado na tarefa, uma vez que são elementos a serem descobertos ou inferidos e nem sempre um problema vai ter só uma. Problemas fechados possuem relações estruturadas relacionando variáveis para que as incógnitas sejam descobertas univocamente. Por outro lado, quando as variáveis não estão estruturadas para haver somente uma solução ou esta dependa do modelo que se adote, o problema pode ser considerado aberto. Neste último, os parâmetros podem ser abordados de distintas maneiras, já que nem todos são dados e alguns devem até ser inferidos, estimados ou considerados impertinentes pelo sujeito (Pantoja \& Moreira, 2019).

Os objetos sempre são pertinentes ou não a uma situação, pois devemos indicalos ou não no problema. Logo, a referência aos objetos é feita via conceitos-em-ação, que têm característica de pertinência. As variáveis têm característica dupla, pois podem ser tanto argumentos e funções proposicionais com valor de verdade ou falsidade quanto podem ou não ser pertinentes ou impertinentes. Portanto, a referência às variáveis é estabelecida por meio de conceitos-em-ação e de teoremas-em-ação. Nesta perspectiva, resolver um problema é encontrar um conjunto de teoremas-em-ação e conceitos-emação descrevendo uma ou mais incógnitas (Pantoja \& Moreira, 2019).

Em situações da teoria eletromagnética, elétrons são objetos. Eles são pertinentes ou não, pois não faz sentido dizer que o elétron é verdadeiro ou falso, eles classificam elementos reais. Por outro lado, dizer que o elétron tem carga elétrica igual a $1,6 \times 10^{-19} \mathrm{C}$ ou que a relação entre densidade de carga elétrica segue a equação de continuidade inclui valor de verdade ou de falsidade. Podemos dizer que ambas são afirmativas verdadeiras do ponto de vista científico, inclusive. Essa é a diferença básica entre teoremas-em-ação e conceitos-em-ação que se reflete nos parâmetros da situação (Pantoja \& Moreira, 2019; Vergnaud, 2013).

O processo de conceitualização pode ser entendido como um vínculo entre um esquema e uma situação. Por um lado, é necessário que a situação tenha informações conceituais identificáveis e representáveis, por outro, é preciso que o sujeito seja capaz de selecionar, relacionar, organizar e aprovisionar estas informações durante a atividade. Em outras palavras, o sujeito precisa mobilizar seus invariantes operatórios para compreender os elencados na situação, o que caracteriza um processo adaptativo do esquema à situação (Vergnaud, 2009). Sintetizamos as ideias apresentadas sobre os parâmetros e as situações na Figura 4. 
Figura 4. Interação entre esquema e situação

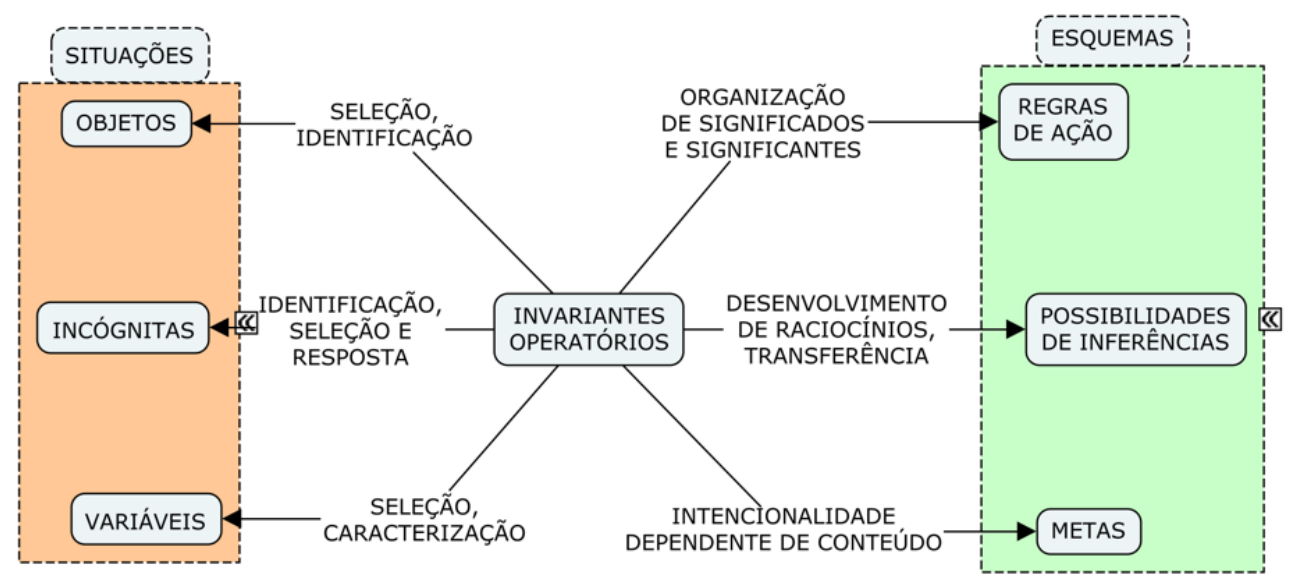

Por fim, existe um deslocamento epistemológico do campo conceitual da indução eletromagnética em relação aos da eletrostática e da magnetostática, em função de a variação temporal dos campos eletromagnéticos dever ser levada em conta (Pantoja \& Moreira, 2019). Neste trabalho, o entendimento do conceito de indução eletromagnética envolve quatro tipos de contextos distintos, nomeadamente, circuitos em movimento em uma região de campo magnético estacionário, criação de campos elétricos no espaço a partir de campos magnéticos variáveis no tempo, produção de campos magnéticos no espaço a partir de campos elétricos variáveis no tempo e geração de ondas eletromagnéticas no espaço. Esses quatro contextos são correlatos, mas a discussão de cada caso demandaria um volume muito grande de conteúdo, sobretudo porque o conceito de campo eletromagnético possui três facetas epistemológicas diferentes, a saber, a de fonte, a de força e a de energia (Nousianen \& Koponen, 2017). Portanto, nesta pesquisa faremos uma abordagem geral sobre o conceito de indução eletromagnética e em outra abordaremos as semelhanças e diferenças entre os casos.

\section{Resultados}

Nos fenômenos de indução eletromagnética, a força eletromagnética entre sistemas de cargas e correntes é descrita pela força de Lorentz e as velocidades são sempre menores que a da luz. O estudo de movimentos acelerados ou a altas velocidades envolve necessariamente o cômputo do tempo retardado, o que permite a existências de efeitos temporais e de movimento. Para este caso, o campo eletromagnético mais simples é o de Lienard-Wiechert para uma carga pontual em movimento arbitrário (Griffiths, 1999). Relacionamos os conceitos mais pertinentes do campo conceitual da eletrodinâmica na Figura 5.

Por outro lado, o campo eletromagnético de uma carga em movimento se reduz ao da carga pontual em movimento lento (próximas da velocidade de deriva em fios condutores) e ao de uma carga elétrica parada quando se fazem "as devidas aproximações", o que implica construir operações linguísticas que "traduzem" dois 
campos epistemológicos incomensuráveis ${ }^{7}$ (Kuhn, 1997). Portanto, a mesma diferença entre campos elétricos e magnéticos estáticos é estabelecida entre os campos elétrico e magnético dinâmicos. Seguimos discutindo as classes primárias $\left(C_{P}\right)$ e secundárias $\left(C_{S}\right)$ de situações.

São apresentadas classes primárias de situações componentes do campo conceitual da indução eletromagnética a seguir:

- descrição de interações eletromagnéticas $(\Sigma)$;

- representação simbólica do campo eletromagnético $(\Theta)$;

- representação analógica do campo eletromagnético $(\Phi)$;

- cálculo de campos eletromagnéticos (T).

Figura 5. Mapa conceitual relacionando os conceitos mais centrais do conteúdo de indução eletromagnética

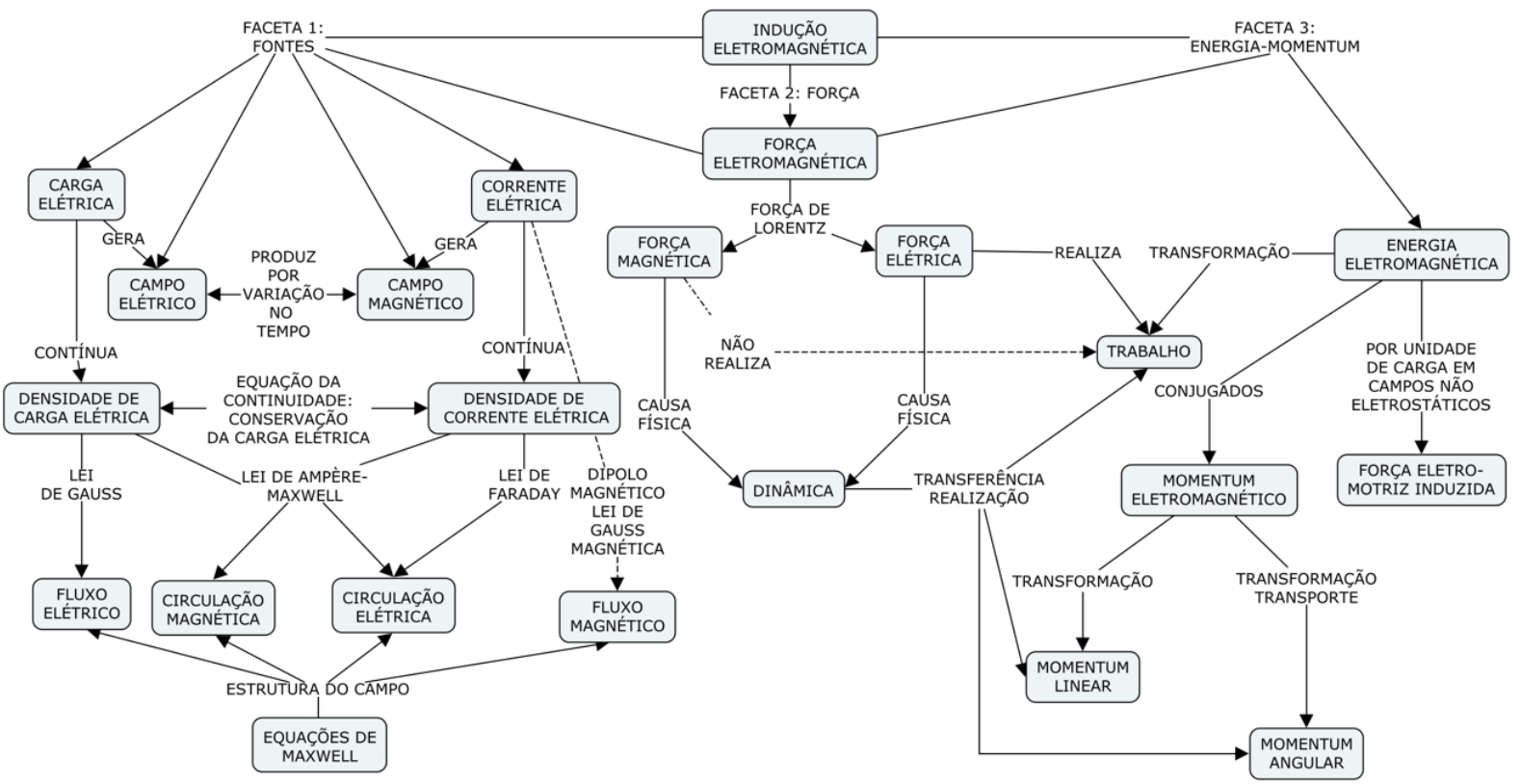

Os conceitos de campo elétrico, campo magnético, força elétrica, força magnética, momentum do campo eletromagnético, energia do campo eletromagnético e torque devido ao campo eletromagnético têm papel central para a resolução de problemas da classe primária de situações $\Sigma$. Para ser resolvida ela requer quatro operações de pensamento gerais, a saber:

1. reconhecimento de fontes de campo eletromagnético;

2. reconhecimento da interação como de origem eletromagnética;

3. aplicação operacional da lei de interação;

4. descrição do estado dinâmico dos objetos deprova interagentes quantitativamente ou qualitativamente.

7 Paradigmas incomensuráveis não podem ser traduzidos um no outro, exceto se em um deles sejam introduzidos novos elementos de linguagem (Kuhn, 1997). 
O reconhecimento de fontes de campo eletromagnéticas requer duas operações de pensamento específicas. A identificação de fontes de campo eletromagnético $\left(\mathrm{A}_{\mathrm{U}}\right)$ inclui o apontamento dos objetos do problema, enquanto a caracterização das fontes de campo eletromagnético ao longo do tempo $\left(B_{U}\right)$ se refere à descrição das variáveis relacionadas a estes objetos. $\mathrm{O}$ subscrito $U$ indica que estas operações de pensamento específicas são aplicadas para todas as outras classes primárias de situações, pois em todas tal ação de reconhecimento de fontes de campo eletromagnético é indispensável. Assim, tudo que está descrito aqui se aplica de forma semelhante para as outras três classes primárias, o que nos furta de discutir este tópico novamente.

O reconhecimento da natureza da interação eletromagnética, solicita, no mínimo, quatro operações de pensamento específicas, a saber. A descrição da relação entre força elétrica e campo eletromagnético $\left(A_{\Sigma}\right)$ e a descrição da relação entre a força magnética e o campo eletromagnético $\left(B_{\Sigma}\right)$ envolvem a diferenciação ou não entre campo e força do ponto de vista operacional. A descrição da natureza ontológica do campo eletromagnético $\left(C_{\Sigma}\right)$ inclui a interpretação onto-epistemológica do campo eletromagnético, enquanto a descrição do papel do campo eletromagnético na interação eletromagnética $\left(D_{\Sigma}\right)$ se propõe a descrever conceitualmente o papel do campo eletromagnético nas interações. Em situações quantitativas, será necessário calcular o campo eletromagnético em questão (há uma classe de situações construída para isto).

A aplicação da lei de interação envolve, minimamente, sete operações de pensamento específicas. A aplicação operacional da lei de força eletromagnética $\left(E_{\Sigma}\right)$ trata do cálculo operacional, qualitativo ou quantitativo, da força eletromagnética. A localização da energia eletromagnética $\left(F_{\Sigma}\right)$ e a localização do momentum eletromagnético $\left(G_{\Sigma}\right)$ relacionam-se à descrição de onde está a energia e o momentum eletromagnéticos (partículas, campo, nos dois). A caracterização da natureza do campo eletromagnético $\left(H_{\Sigma}\right)$ diz respeito à designação conceitual do campo eletromagnético ${ }^{8}$. Já a descrição das trocas de energia eletromagnética $\left(I_{\Sigma}\right)$ e a descrição das trocas de momentum eletromagnético $\left(J_{\Sigma}\right)$ referem-se à representação da interação eletromagnética por meio de trocas relativas a essas duas grandezas 9 . A aplicação do princípio da superposição $\left(K_{\Sigma}\right)$ inclui a utilização conceitual e operacional desse princípio. Embora seja possível abordar $H_{\Sigma}$ e $I_{\Sigma}$ juntas, optou-se por separa-los para facilitar a caracterização de possíveis invariantes operatórios.

Para a descrição da evolução temporal do estado dinâmico dos objetos interagentes quantitativamente ou qualitativamente requer duas operações de pensamento a esquematização da força resultante sobre objetos de prova $\left(A_{M}\right)$ e a descrição da dinâmica dos objetos de prova $\left(B_{M}\right)$. Tais ações dependem de conhecimentos do campo conceitual da mecânica clássica ${ }^{10}$, em especial da dinâmica. O programa de pesquisa de Lorentz

8 Se eletrostático, não-eletrostático ou eletrodinâmico. A $h_{G} 4$ nos permite perceber se o aluno consegue diferenciar os quatro contextos da eletrodinâmica mencionados na introdução.

9 se mediadas pelo campo, ou realizada diretamente pelas partículas.

10 Os símbolos $M, G, A, V$ querem dizer, respectivamente, mecânica clássica, geometria, álgebra/análise, análise vetorial. 
permite a relação entre a dinâmica clássica, endossada pela escola de ação à distância, e o eletromagnetismo campista. A Figura 6 arrola as $h_{G}$ e as $h_{S}$ necessárias para lidar com esse tipo de situação.

Figura 6. Relação entre operações de pensamento gerais e específicas para a classe primária de situações descrição de interações eletromagnéticas $(\Sigma)$

\begin{tabular}{|c|c|l|}
\hline $\mathbf{h}_{\mathbf{G}}$ & $\mathbf{h}_{\mathrm{S}}$ & Descrição \\
\hline \multirow{4}{*}{1} & $A_{U}$ & Identificação de fontes do campo eletromagnético \\
\cline { 2 - 3 } & $B_{U}$ & Caracterização das fontes de campo eletromagnético ao longo do tempo \\
\hline \multirow{4}{*}{2} & $A_{\Sigma}$ & Descrição da relação entre força elétrica e campo eletromagnético \\
\cline { 2 - 3 } & $B_{\Sigma}$ & Descrição da relação entre a força magnética e o campo eletromagnético \\
\cline { 2 - 3 } & $C_{\Sigma}$ & Descrição da natureza ontológica do campo eletromagnético \\
\cline { 2 - 3 } & $D_{\Sigma}$ & Descrição do papel do campo eletromagnético na interação eletromagnética \\
\hline \multirow{4}{*}{3} & $E_{\Sigma}$ & Aplicação operacional da lei de força eletromagnética \\
\hline \multirow{4}{*}{4} & $F_{\Sigma}$ & Localização da energia eletromagnética \\
\cline { 2 - 3 } & $G_{\Sigma}$ & Localização do momentum eletromagnético \\
\cline { 2 - 3 } & $H_{\Sigma}$ & Caracterização da natureza do campo eletromagnético \\
\cline { 2 - 3 } & $I_{\Sigma}$ & Descrição das trocas de energia eletromagnética \\
\hline & $J_{\Sigma}$ & Descrição das trocas de momentum eletromagnético \\
\cline { 2 - 3 } & $K_{\Sigma}$ & Aplicação do princípio da superposição \\
\hline \multirow{2}{*}{4} & $A_{M}$ & Esquematização da força resultante sobre objetos de prova \\
\cline { 2 - 3 } & $B_{M}$ & Descrição da dinâmica dos objetos de prova \\
\hline
\end{tabular}

Construímos quatro classes de situações secundárias discutidas e exemplificadas a seguir. São elas, a descrição de interações eletromagnéticas entre um portador pontual de carga elétrica e:

- outro portador de carga pontual em movimento arbitrário $\left(\Sigma_{\mathrm{A}}\right)$;

- vários portadores pontuais de cargas elétricas em movimento arbitrário $\left(\Sigma_{\mathrm{B}}\right)$;

- sistemas de cargas e correntes elétricas distribuídos continuamente, de forma conhecida a priori e variável no tempo $\left(\Sigma_{\mathrm{C}}\right)$;

- sistemas de cargas e correntes elétricas distribuídos continuamente, de forma desconhecida a priori e variável no tempo $\left(\Sigma_{\mathrm{D}}\right)$.

Na classe secundária de situações $\Sigma_{A}$, temos complicações impostas pelo tempo de retardo dos campos eletromagnéticos produzidos pelas cargas elétricas cujos portadores estão em movimento arbitrário. Tal situação não ocorre na magnetostática e nem na eletrostática (Griffiths, 1999). Desta forma, os objetos dessa classe secundária de situações são dois portadores pontuais de carga elétrica em movimento arbitrário interagindo via campo eletromagnético. Como variáveis possíveis temos cargas elétricas, posições das partículas com respeito a um referencial inercial $(S)$, distância entre as partículas, velocidade das partículas com respeito a $S$, velocidade relativa entre as partículas, aceleração das partículas com respeito a $S$, aceleração relativa das partículas, campo elétrico, campo magnético, tempo, tempo de retardo, força elétrica, força magnética, 
além de grandezas derivadas como momentum linear, momentum angular, energia cinética, energia eletromagnética, etc.

A expressão para o campo eletromagnético produzido por uma carga em movimento arbitrário é dada pelos chamados campos de Lièrnard-Wiechert ${ }^{11}$ (Griffiths, 1999), que, em geral, não é discutida em cursos de Física básica, mas em cursos de eletromagnetismo de ciclo profissional, pois envolve matemática avançada. As situações mais trabalhadas deste campo conceitual em livros didáticos pertencem à classe $\Sigma_{C}$ que discutiremos posteriormente.

Suponha um portador de carga elétrica pontual, $\mathrm{P}$, movendo-se com velocidade velocidade $\vec{v}_{P}(t)=v_{o} \hat{x}$ na mesma direção que um portador de carga elétrica, $\mathrm{F}$, que se move a velocidade $\vec{v}_{F}(t)=\left(v_{0}+a t\right) \hat{x}$. Os portadores de carga estão a uma distância $d$ em $t=0$. Qual a força eletromagnética experimentada por $\mathrm{P}$ ao longo do tempo?

Os objetos são os dois portadores de carga elétrica P e F. As variáveis são as cargas elétricas de $\mathrm{P}$ e de $\mathrm{F}$, as velocidades de $\mathrm{P}$ e de $\mathrm{F}$, a distância entre $\mathrm{P}$ e $\mathrm{F}$, além do tempo de retardo do campo eletromagnético. A incógnita é o vetor força eletromagnética, determinado pela força de Lorentz. As operações de pensamento mais prováveis de serem explicitadas no processo de resolução do problema são $A_{U^{*}} B_{U^{*}} A_{\Sigma^{\prime}} B_{\Sigma^{\prime}} E_{\Sigma^{\prime}} A_{M^{*}}$ As duas primeiras devem ser realizadas de maneira consciente, pois envolvem a referência aos objetos e variáveis. $\mathrm{O}$ mesmo se aplica a $A_{\Sigma}, B_{\Sigma} \mathrm{e} E_{\Sigma}$, porque se referem às variáveis a serem manipuladas para alcançar a solução e à incógnita do problema, a força eletromagnética. $A_{M}$ surge para a determinação desta força, mas a alteração da incógnita para "descreva as trocas de energia entre esses portadores de carga elétrica" ativaria outras operações de pensamento que, de outra forma, podem ficar implícitas e inconscientes.

A classe $\Sigma_{B}$ inclui problemas parecidos com os da classe secundária de situações $\Sigma_{A}$, porém surgem algumas complicações impostas pelas posições das cargas que produzem os campos eletromagnéticos, pois elas estão a diferentes distâncias e terão tempos de retardo distintos, o que torna essas situações significativamente mais abstratas do que situações correlatas dos campos conceituais dos conceitos da magnetostática (Pantoja \& Moreira, 2020) e da eletrostática (Pantoja \& Moreira, 2019). Ademais, existe um número maior de objetos e as variáveis incluem momento de dipolo elétrico, força elétrica resultante, força magnética resultante, campo elétrico resultante e campo magnético resultante, além das possivelmente presentes nas situações $\Sigma_{A}$.

11

O campo elétrico é dado por

$$
\vec{E}(\vec{r}, t)=\frac{1}{4 \pi \epsilon_{0}} \frac{q}{r^{2} c^{2}\left[\hat{r} \cdot\left(\hat{r}-\frac{\vec{v}}{c}\right)\right]^{3}}\left[\left(c^{2}-v^{2}\right)\left(\hat{r}-\frac{\vec{v}}{c}\right)+\hat{r} \times\left(\hat{r}-\frac{\vec{v}}{c}\right) \times \vec{a}\right]
$$

e campo magnético por $\vec{B}(\vec{r}, t)=\frac{1}{c} \hat{r} \times \vec{E}(\vec{r}, t)$. 
Suponha dois portadores de cargas pontuais $F$ e $G$ movendo-se, respectivamente, com $\vec{v}_{F}(t)=\left(v_{0}+a t\right) \hat{x}$ e $\vec{v}_{G}(t)=\left(v_{0}+a t\right) \hat{y}$. Os portadores de carga elétrica estão inicialmente em $(0,0,0)$ e um portador de carga elétrica pontual $P$ está em $(0,0, d)$. Qual a equação de movimento seguida por $P$ ao longo do tempo?

Os objetos são os portadores de carga elétrica F, G e P. As variáveis são as cargas elétricas dos três portadores, suas velocidades e suas posições, além do tempo de retardo. A incógnita é uma equação de movimento seguida por $\mathrm{P}$, ou seja, a determinação da posição como função do tempo. Aqui há menção explícita à equação de movimento seguida pelo objeto de prova, diferentemente do exemplo para $\Sigma_{A}$. As operações de pensamento específicas a serem processadas conscientemente incluem também $\Sigma_{\Sigma}$ e $B_{M}$, pois é necessário usar o princípio da superposição e determinar a dinâmica do objeto de prova, encontrando sua equação de movimento. Desta forma, a maneira de apresentar as incógnitas também influenciam na conceitualização.

A classe $\Sigma_{C}$ inclui novas variáveis além das possíveis para as classes primárias $\Sigma_{A}$ e $\Sigma_{B}$, tais como a densidade de carga elétrica e a densidade de corrente elétrica que mudam espaço-temporalmente, resistência elétrica, capacitância elétrica e indutância magnética, pois envolvem sistemas de portadores de carga elétrica e condutores de corrente elétrica distribuídos continuamente como novos objetos. Ademais, abrese possibilidade de discussão de eletromagnetismo na matéria, além da inclusão de variáveis ou incógnitas como a polarização e a magnetização, por haver possibilidade de haver distribuição contínua de momentos de dipolo elétrico e magnético. Há que se incluir neste rol a força magnética, a força elétrica, o campo magnético e o campo elétrico resultantes computados por operações de integração, além do conceito de força eletromotriz induzida, que nos possibilita levar em conta as trocas de energia entre campos e circuitos elétricos. $\mathrm{O}$ exemplo abaixo envolve um caso comumente abordado em cursos de Física de ciclo básico.

Suponha um elétron posicionado no interior de um solenoide de raio $R$ e que a corrente deste aparato diminua a uma taxa constante de modo que seu campo magnético seja reduzido de 10,7 mT/s. Qual a aceleração instantânea (direção, módulo e sentido) experimentada pelo elétron quando em um ponto distante de $\mathrm{R} / 2$ do centro do solenoide? Suponha $R=4,82 \mathrm{~cm}$ (o espalhamento que ocorre além de $R$ não mudará a responsa na medida em que existe uma simetria axial em relação à perpendicular ao eixo axial). (Adaptado de Halliday et al., 2006).

Os objetos são o elétron e o solenoide condutor de corrente elétrica. As variáveis são a carga elétrica do elétron, a taxa de variação do campo magnético no interior do solenoide e o raio do solenoide. A incógnita é a aceleração do elétron a uma distância $R / 2$ do solenoide. Este é um caso particular em que a resolução de um problema $\Sigma_{C}$ é matematicamente mais simples que os das classes $\Sigma_{A}$ e $\Sigma_{B}$, pois a determinação do campo elétrico é feita por meio da lei de Faraday na forma integral. As operações de 
pensamento são basicamente as mesmas do exemplo, mas a fica implícita na lei de Faraday. A mudança da pergunta para um enunciado do tipo "a energia eletromagnética e o momentum eletromagnético estão no campo, no elétron e no solenoide ou nos dois?” recrutaria explicitamente operações de pensamento $F_{\Sigma}$ e $G_{\Sigma}$, por exemplo.

A classe $\Sigma_{D}$ envolve situações incluindo sistemas de portadores de carga elétrica e condutores de corrente cuja distribuição contínua é desconhecida a priori. As novas variáveis incluídas são o potencial escalar e o potencial vetor, bem como as condições de contorno para os campos e para os potenciais eletromagnéticos. Em geral, é abordada em cursos avançados de óptica ou usada para abordagens generalistas de eletromagnetismo avançado, tal como a determinação da solução dos potenciais e campos retardados.

Qual a aceleração de um elétron colocado no interior de uma guia de ondas retangular?

Este problema tem objetos definidos, mas é aberto, pois suas variáveis precisam ser definidas pelo sujeito. As variáveis incluem o formato retangular da seção transversal da guia de ondas, as condições de contorno sobre este sistema (definidas pelo indivíduo), campo elétrico, campo magnético, força elétrica, forma magnética, tempo, tempo de retardo, pontos do espaço. A incógnita é a aceleração do elétron. A Figura 7 sintetiza os parâmetros mais comuns de cada uma das classes de situações secundárias propostas.

Figura 7. Classificação secundária de situações da classe $\Sigma$ baseada nos parâmetros

\begin{tabular}{|c|l|}
\hline $\mathrm{C}_{\mathrm{s}}$ & Parâmetros \\
\hline$\Sigma_{A}$ & $\begin{array}{l}\text { Portadores pontuais de carga em movimento arbitrário, carga elétrica, posição, distância (prova- } \\
\text { fonte), velocidade, aceleração, trajetória, força elétrica, força magnética, momento de dipolo } \\
\text { magnético, energia eletromagnética, momentum eletromagnético, energia cinética, momentum } \\
\text { linear, momentum angular, campo elétrico (dinâmico), campo magnético (dinâmico), taxa de } \\
\text { variação temporal do campo elétrico e taxa de variação temporal do campo magnético. }\end{array}$ \\
\hline$\Sigma_{B}$ & $\begin{array}{l}\text { Todos os parâmetros possíveis para } \Gamma_{A} \text { mais distribuições discretas de portadores de carga } \\
\text { pontuais, momento de dipolo elétrico, força magnética resultante (soma), força elétrica resultante } \\
\text { (soma), campo elétrico resultante (soma) campo magnético resultante (soma). }\end{array}$ \\
\hline$\Sigma_{C}$ & $\begin{array}{l}\text { Todos os parâmetros possíveis para } \Gamma_{B} \text { mais distribuições contínuas conhecidas de portadores } \\
\text { de cargas elétricas, densidade de carga elétrica, densidade de corrente elétrica, resistência, } \\
\text { capacitância, indutância, polarização, magnetização, deslocamento elétrico, indução magnética, } \\
\text { força elétrica resultante (integral), força magnética resultante (integral), campo elétrico resultante } \\
\text { (integral), campo magnético resultante (integral) e força eletromotriz induzida. }\end{array}$ \\
\hline$\Sigma_{D}$ & $\begin{array}{l}\text { Todososparâmetros possíveis para } \Gamma_{C} \text { mais distribuições contínuas desconhecidase determináveis } \\
\text { de portadores de cargas elétricas, distribuições contínuas desconhecidas e determináveis de } \\
\text { portadores de correntes elétricas, condições de contorno para o campo magnético e para o } \\
\text { campo elétrico e para o campo de indução magnética, potencial escalar, potencial vetor. }\end{array}$ \\
\hline
\end{tabular}

As dificuldades associadas a este problema não são somente matemáticas, tal como a de resolver uma equação diferencial parcial, mas também são físicas. Por exemplo, o princípio da superposição está implícito na equação diferencial que traz como variáveis os campos elétrico e magnético. A determinação das condições de contorno 
requer relações aprofundadas entre suposições físicas e construções matemáticas. Isto mostra que esta tarefa não envolve somente um esquema para descrever a interação eletromagnética, mas um para calcular o campo eletromagnético.

As situações nas quais se deve construir representação simbólica do campo eletromagnético $(\Theta)$ e representação analógica do campo eletromagnético $(\Phi)$ incluem cinco operações de pensamento primárias para serem dominadas, a saber:

1. reconhecimento de fontes de campo magnético;

2. reconhecimento de aspectos algébrico-analítico $(\Theta)$ ou geométricos $(\Phi)$ das fontes;

3. localização de pontos do espaço;

4. mapeamento do significado das equações de campo eletromagnético em aspectos simbólico-relacionais $(\Theta)$ ou em aspectos geométricos $(\Phi)$;

5. associação de vetores ou escalares a pontos do espaço para estabelecer a representação simbólica ou analógica

Após o reconhecimento de fontes de campo eletromagnético, é necessário apontar aspectos geométricos $(\Phi)$ ou algébricos-analíticos $(\Theta)$ das fontes e suas variações ao longo do tempo. Esta operação de pensamento geral está fundada essencialmente nos campos conceituais da geometria $(\Phi)$ e da álgebra/análise $(\Theta)$, o que torna estas duas classes distintas, embora necessitem de ideias correlatas. As operações de pensamento específicas para resolver tarefas desta classe são a identificação de aspectos geométricos $\left(A_{G}\right.$, para situações $\left.\Phi\right)$ ou algébrico-analíticos das fontes $\left(A_{A}\right.$, para situações $\left.\Theta\right)$. A primeira se refere à enumeração das características relevantes para a identificação das fontes, por exemplo, fazer referência a uma espira de corrente, enquanto a segunda se refere à atribuição de características às fontes em si, tal como descrever sua circunferência, sua forma geométrica, a taxa de variação temporal das suas dimensões ou da carga elétrica/ corrente elétrica conduzida etc.

Também é indispensável a localização de pontos do espaço, pois a magnitude e a orientação do campo diferirão conforme mudem os pontos relevantes do espaço e passe o tempo, assim como os valores de fluxo magnético, de fluxo elétrico, de circulação magnética e circulação elétrica. Tal operação de pensamento geral é composta por outras três específicas autoexplicativas, quais sejam, a determinação de posições de pontos do espaço $\left(A_{V}\right)$, a determinação de posições de fontes ao longo do tempo $\left(B_{V}\right)$ e a determinação de distâncias entre ponto e fontes ao longo do tempo $\left(C_{V}\right)$. Os pontos do espaço são considerados constantes e a posição da fonte é que muda ao longo do tempo em situações desse tipo. As operações de pensamento específicas podem ser embasadas em álgebra-análise $(\Theta)$ ou em geometria $(\Phi)$,

Em quarto lugar, é imprescindível para o aluno empregar o mapeamento do significado das equações de campo eletromagnético em aspectos simbólico-relacionais $(\Theta)$ ou em aspectos geométricos $(\Phi)$ na situação. Esta operação de pensamento geral pode ser decomposta em outras quatro específicas servindo a ambas às classes primárias, a saber, a interpretação física do fluxo elétrico $\left(A_{\Phi}\right.$ e $\left.A_{\theta}\right)$, a interpretação física do fluxo magnético 
$\left(B_{\Phi}\right.$ e $\left.B_{\theta}\right)$, a interpretação física da circulação elétrica $\left(C_{\Phi}\right.$ e $\left.C_{\theta}\right)$ e a interpretação física da circulação magnética $\left(D_{\Phi}\right.$ e $\left.D_{\theta}\right)$. Esta operação de pensamento geral é importante, pois o conhecimento completo do campo eletromagnético envolve a determinação do fluxo ou do divergente e da circulação ou do rotacional de ambos os campos. Estes campos estão acoplados por derivadas temporais parciais, logo desenvolver interpretação das equações estruturantes é imprescindível para completar a representação, seja ela simbólica ou analógica.

Em quinto lugar, prossegue-se ao estabelecimento da representação. Esta operação de pensamento geral é destrinchada em nove operações de pensamento específicas, nomeadamente, a relação entre o fluxo elétrico e campo elétrico $\left(E_{\Phi}\right.$ e $\left.E_{\theta}\right)$, a relação entre fluxo magnético e campo elétrico $\left(F_{\Phi}\right.$ e $\left.F_{\theta}\right)$, a relação entre a circulação elétrica e campo elétrico $\left(G_{\Phi}\right.$ e $\left.G_{\theta}\right)$, a relação entre o fluxo elétrico e campo elétrico $\left(H_{\Phi}\right.$ e $\left.H_{\theta}\right)$, a relação entre o fluxo elétrico e campo magnético $\left(I_{\Phi}\right.$ e $\left.I_{\theta}\right)$, a relação entre fluxo magnético e campo magnético $\left(J_{\Phi} \mathrm{e} J_{\theta}\right)$, a relação entre circulação elétrica e campo magnético $\left(K_{\Phi} \mathrm{e} K_{\theta}\right)$, a relação entre o fluxo elétrico e o campo magnético $\left(L_{\phi}\right.$ e $\left.L_{\oplus}\right)$, o estabelecimento da representação analógica $\left(C_{G}\right)$ ou simbólica $\left(C_{A}\right)$. Esta última dependerá de aspectos geométricos ou algébricos em função da relação estabelecida. Vale lembrar que as equações de campo eletromagnético já trazem consigo o princípio da superposição de maneira implícita. A Figura 8 relaciona as $h_{S}$ e as $h_{G}$ componentes dessas duas classes de situações.

Embora estas classes primárias de situação aparentem ser iguais, as operações de pensamento necessárias para construir estas distintas representações simbólicas ou analógicas são em base diferentes, o que nos conduz a coloca-las em distintos grupos (Pantoja \& Moreira, 2019). A argumentação é simples, mas empiricamente factível - há pessoas com maior tendência à representação simbólica, enquanto outras que tendem mais à representação analógica. Portanto, estas competências devem ser diferentes e é papel do professor realizar a mediação para integrar as duas competências.

Distinguimos as quatro classes secundárias de situações a seguir. São elas, a representação analógica ou simbólica em pontos do espaço do campo eletromagnético devido a:

- um portador pontual de carga elétrica em movimento arbitrário $\left(\Phi_{\mathrm{A}}\right.$ ou $\left.\Theta_{\mathrm{A}}\right)$;

- um conjunto discreto de portadores pontuais de cargas elétricas em movimento arbitrário $\left(\Phi_{\mathrm{B}}\right.$ ou $\left.\Theta_{\mathrm{B}}\right)$;

- um sistema de cargas e correntes elétricas distribuído continuamente, de forma conhecida a priori e variável ao longo do tempo $\left(\Phi_{\mathrm{C}}\right.$ ou $\left.\Theta_{\mathrm{C}}\right)$;

- um sistema de cargas e correntes elétricas distribuído continuamente, de forma desconhecida a priori e variável ao longo do tempo $\left(\Phi_{\mathrm{D}}\right.$ ou $\left.\Theta_{\mathrm{D}}\right)$. 
Figura 8. Relação entre operações de pensamento gerais e específicas para as classes primárias de situações representação analógica do campo eletromagnético $(\Phi)$ e representação simbólica do campo eletromagnético $(\Theta)$

\begin{tabular}{|c|c|c|c|}
\hline$h_{p}$ & \multicolumn{2}{|c|}{$\mathbf{h}_{\mathrm{s}}$} & Descrição \\
\hline \multirow{2}{*}{1} & \multicolumn{2}{|c|}{$A_{U}$} & Identificação de fontes do campo magnético \\
\hline & \multicolumn{2}{|c|}{$B_{\mathrm{U}}$} & Caracterização das fontes de campo magnético \\
\hline \multirow[b]{2}{*}{2} & $A_{\mathrm{G}}$ & $A_{\mathrm{A}}$ & Identificação de aspectos geométricos $(\Phi)$ ou algébrico-analíticos $(\Theta)$ das fontes \\
\hline & $B_{\mathrm{G}}$ & $B_{\mathrm{A}}$ & $\begin{array}{l}\text { Caracterização de aspectos geométricos }(\Phi) \text { ou algébrico-analíticos }(\Theta) \text { das } \\
\text { fontes }\end{array}$ \\
\hline \multirow{3}{*}{3} & \multicolumn{2}{|c|}{$A_{\mathrm{V}}$} & Determinação de posições de pontos do espaço \\
\hline & \multicolumn{2}{|c|}{$B_{\mathrm{V}}$} & Determinação de posições de fontes ao longo do tempo \\
\hline & \multicolumn{2}{|c|}{$C_{\mathrm{V}}$} & Determinação de distâncias entre ponto e fontes ao longo do tempo \\
\hline \multirow{4}{*}{4} & $A_{\phi}$ & $A_{\Theta}$ & Interpretação física do fluxo elétrico \\
\hline & $B_{\phi}$ & $B_{\Theta}$ & Interpretação física do fluxo magnético \\
\hline & $C_{\phi}$ & $C_{\Theta}$ & Interpretação física da circulação elétrica \\
\hline & $D_{\Phi}$ & $D_{\Theta}$ & Interpretação física da circulação magnética \\
\hline \multirow{9}{*}{5} & $E_{\Phi}$ & $E_{\Theta}$ & Relação entre o fluxo elétrico e campo elétrico \\
\hline & $F_{\phi}$ & $F_{\Theta}$ & Relação entre fluxo magnético e campo elétrico \\
\hline & $G_{\phi}$ & $G_{\Theta}$ & Relação entre a circulação elétrica e campo elétrico \\
\hline & $H_{\Phi}$ & $H_{\Theta}$ & Relação entre o fluxo elétrico e campo elétrico \\
\hline & $I_{\Phi}$ & $I_{\Theta}$ & Relação entre o fluxo elétrico e campo magnético \\
\hline & $J_{\Phi}$ & $J_{\Theta}$ & Relação entre fluxo magnético e campo magnético \\
\hline & $K_{\Phi}$ & $K_{\Theta}$ & Relação entre circulação elétrica e campo magnético \\
\hline & $L_{\Phi}$ & $L_{\Theta}$ & Relação entre o fluxo elétrico e o campo magnético \\
\hline & $C_{G}$ & $C_{A}$ & Estabelecimento da representação analógica $(\Phi)$ ou simbólica $(\Theta)$ \\
\hline
\end{tabular}

As classes primárias $\Theta$ e $\Phi$ envolvem operações de pensamento distintas, embora possam incluir os mesmos objetos e variáveis. Isto implica em formas diferentes de propor as incógnitas. Diferenciamos as duas através da proposição de uma incógnita diferente para cada uma nos exemplos apresentados.

Nas classes secundárias de situações $\Phi_{\mathrm{A}}$ e $\Theta_{\mathrm{A}}$ sempre haverá um objeto em questão, que é um portador pontual de carga elétrica em movimento arbitrário no espaço. Assim, as variáveis podem envolver carga elétrica, posição, velocidade, aceleração, momento magnético, campo elétrico, campo magnético, fluxo elétrico, fluxo magnético, circulação elétrica e circulação magnética arbitrariamente variáveis no tempo, além de curvas amperianas, superfícies gaussianas ${ }^{12}$, aspectos geométricos $(\Phi)$, aspectos algébricoanalíticos $(\Theta)$, tempo de retardo e tempo. Apresentamos exemplos a seguir.

12 Utilizamos a denominação amperiana para qualquer curva fechada orientada, seja na lei de Faraday ou na lei de Ampère-Maxwell. Tal denominação foi feita a mérito de síntese. 
Suponha um portador de carga pontual, F, movendo-se com velocidade $\vec{v}_{F}(t)=\left(v_{0}+a t\right) \hat{x}$. F está distante de $d$ de um ponto $P$ (ao longo do eixo $x$ ) em $\mathrm{t}=$ 0 . Como você descreveria o balanço de energia eletromagnético no ponto $P$ ao longo do tempo?

Os objetos são um portador de carga elétrica $\mathrm{F}$ e um ponto do espaço. As variáveis são a carga elétrica de F, a velocidades de F, além da distância entre P e F. A incógnita é a energia do campo eletromagnético no ponto $\mathrm{P}$ em um instante $t$. Já temos mudança da classe primária de situações $\Sigma$ para $\Theta$ por duas razões, quais sejam, o trabalho com campos e não com forças e o tipo de incógnita solicitado. Em primeiro lugar, o balanço energético envolve o trabalho mecânico realizado para acelerar as cargas de fonte, a variação da energia eletromagnética em um ponto do espaço e o divergente do vetor de Poynting. É uma relação intricada entre campo eletromagnético e energia que envolve as quatro equações de Maxwell $\left(\mathrm{A}_{\Theta}, B_{\Theta}, C_{\Theta}, D_{\Theta}\right)$. Também é necessário interpretá-las $\left(E_{\Theta}\right.$ $\left.-L_{\Theta}\right)$, bem como identificar as fontes e o tipo de campo que produzem $\left(A_{U}, B_{U}\right)$. Como a relação é de tipo abstrata, podemos caracteriza-la como representação simbólica.

Suponha um portador de carga pontual, $F$, movendo-se com velocidade $\vec{v}_{F}(t)=\left(v_{0}+a t\right) \hat{x}$. F está distante de $d$ de um ponto $P$ (ao longo do eixo $x$ ) em $t$ $=0$. Que forma teria um esboço qualitativo dos vetores campo elétrico e campo magnético no ponto $P$ ao longo do tempo?

Os objetos e variáveis são os mesmos da situação $\Theta_{A}$, mas dessa vez a incógnita é um diagrama de setas para os campos elétrico e magnético no ponto $\mathrm{P}$ em diferentes instantes de tempo. Podemos perceber que a simples mudança de incógnita já solicita outras operações de pensamento secundárias, mas voltada à representação espaçovisualmente análoga em estrutura ao real $\left(A_{\mathrm{G}}, B_{\mathrm{G}}, C_{\mathrm{G}}\right)$.

As classes $\Phi_{B}$ e $\Theta_{B}$ têm como objetos dois ou mais portadores de carga elétrica pontual em movimento arbitrário no espaço. Elas envolvem todas as variáveis das classes $\Phi_{\mathrm{A}} \mathrm{e} \Theta_{\mathrm{A}} \mathrm{e}$, além de introduzirem novas, tais como o campo elétrico, o campo magnético, a força elétrica e a força magnética resultantes.

Suponha dois portadores de cargas pontuais $F$ e $G$ movendo-se, respectivamente, com $\vec{v}_{F}(t)=\left(v_{0}+a t\right) \hat{x}$ e $\vec{v}_{G}(t)=\left(v_{0}+a t\right) \hat{y}$. Os portadores de carga elétrica estão inicialmente em $(0,0,0)$. Suponha uma curva amperiana circular no plano, que informações podemos obter a partir da determinação da circulação do campo elétrico e do campo magnético?

Os objetos são os portadores de carga elétrica F, G e P. As variáveis são as cargas elétricas dos três portadores, suas velocidades e suas posições, tempo de retardo, curvas amperianas para cálculo das circulações elétrica e magnética. Desta vez, a incógnita são valores estimados qualitativamente para a circulação e para o fluxo magnético. Destacamos que a introdução de mais um portador de carga elétrica de fonte requer 
explicitamente agregar o princípio da superposição ${ }^{13}$ na interpretação das equações de campo eletromagnético e na relação destas com o último. A operação de pensamento $C_{A}$ deve ser feita em função do campo eletromagnético resultante devido a todas as fontes do problema.

Suponha dois portadores de cargas pontuais $F$ e $G$ movendo-se, respectivamente, $\operatorname{com} \vec{v}_{F}(t)=\left(v_{0}+a t\right) \hat{x} \quad$ e $\vec{v}_{G}(t)=\left(v_{0}+a t\right) \hat{y}$. Os portadores de carga elétrica estão inicialmente em $(0,0,0)$. Que formato aproximado terão as linhas de campo elétrico resultante para os instantes $t=0 ; t=\frac{\pi}{2} ; t=\pi ; t=\frac{3 \pi}{2}$ ?

Os objetos e variáveis são os mesmos da situação $\Theta_{B}$, mas a incógnita se dirige a diagramas de linhas de campo elétrico para os instantes de tempo solicitados. Aqui vale o mesmo caso já discutido para a diferença entre $\Phi_{B}$ e $\Theta_{B}$, mas simplifica a questão propondo os instantes de tempo, o que não era feito na situação exemplificando $\Phi_{B}$.

As classes $\Phi_{C}$ e $\Theta_{C}$ incluem como objetos sistemas de portadores de carga elétrica em movimento arbitrário distribuídos continuamente no espaço e modificáveis com o tempo. Elas envolvem como variáveis as situações das classes $\Phi_{B}$ e $\Theta_{B}$ e envolvem, além das mencionadas, campos elétricos, campos magnéticos, forças elétricas e forças magnéticas resultantes computados na forma de integrais. Há substituição de distribuições discretas por distribuições contínuas de portadores de carga em movimento arbitrário, o que evoca os conceitos de densidade de carga elétrica e de densidade de corrente elétrica modificáveis no espaço e no tempo. Há abertura para introdução de questões sobre eletromagnetismo na matéria, em virtude de se abordar a distribuição contínua de momentos de dipolo elétrico e de dipolo magnético. Isto torna possível abordar os conceitos de indução magnética, deslocamento elétrico, polarização e magnetização para caracterizar variáveis.

Que interpretação qualitativa você estabeleceria para as equações de Jefimenko? Que relação estabeleceria com os conceitos de densidade de carga elétrica, densidade de corrente elétrica, fluxo e circulação?

Este problema é aberto, portanto os objetos devem ser enumerados pelo indivíduo. As variáveis podem incluir densidade de carga elétrica, densidade de corrente elétrica, campo elétrico, campo magnético, tempo, tempo de retardo, fluxo elétrico, fluxo magnético, circulação elétrica e circulação magnética. A incógnita envolve o estabelecimento de relações entre os conceitos. As equações de Jefimenko são soluções particulares para as equações de Maxwell e a elas pode ser somada qualquer solução da equação de ondas homogênea. A nosso ver, isto é suficiente para mostrar que as classes secundárias de tipo $C$ são mais difíceis que as dos tipos $A$ e $B$, pois elas envolvem a noção de distribuição contínua de carga elétrica e necessariamente incluem a passagem do caso discreto para o caso contínuo como obstáculo epistemológico. Não só porque utilizamos integrais, mas porque esta transição é difícil de ser feita sem o domínio conceitual da

13 É um teorema científico que pode ser encontrado na forma teorema-em-ação na conceitualização de muitos alunos. É papel do professor prover condições para que os alunos o explicitem. 
noção matemática de infinitésimo, relacionada fisicamente a distribuições contínuas.

Esboce as linhas de campo elétrico e de campo magnético de uma onda plana eletromagnética para instantes de tempo que achar interessante.

Este problema também é aberto, portanto as fontes de eletromagnético devem ser supostas pelo indivíduo e devem satisfazer a condição de produção de onda plana. As variáveis adotadas podem ser campo elétrico, campo magnético, direção de propagação, pontos do espaço, circulação elétrica, circulação magnética, fluxo elétrico, fluxo magnético e tempo. As incógnitas são diagramas de linhas de campo eletromagnético. Assim como a situação anterior, todas as operações de pensamento são acessíveis e os alunos precisam construir uma explicação ligando as fontes (1) e seus aspectos geométricos (2), pontos do espaço (3), o conteúdo das equações de Maxwell (4) e sua relação com os campos elétrico e magnético (5) para estabelecer a representação analógica.

As classes $\Phi_{D}$ e $\Theta_{D}$ são caracterizadas pela a substituição de distribuições contínuas conhecidas de cargas e correntes elétricas por distribuições desconhecidas a priori. Há a consideração das condições de contorno sobre o campo elétrico e sobre o campo magnético para a constituição de novos conceitos-em-ação e novos teoremasem-ação. Estas classes incluem todos as variáveis possíveis para $\Phi_{C} \mathrm{e} \Theta_{C}$.

Apresente argumentos qualitativos sobre a nulidade do fluxo magnético no interior de uma guia de ondas circular.

Este problema envolve como objetos uma guia de ondas e pontos no seu interior. As variáveis incluem o formato circular da sua seção transversal, campo elétrico, campo magnético, fluxo magnético, circulação magnética, fluxo elétrico, circulação elétrica, tempo, tempo de retardo, pontos do espaço. A incógnita envolve uma cadeia de relações conceituais com a lei de Gauss do magnetismo para explicar a razão de o fluxo ser nulo.

Construa um diagrama de setas do campo elétrico produzido no interior de uma guia de ondas circular.

Este problema envolve objetos e variáveis semelhantes ao da classe $\Theta_{D}$, mas a incógnita envolve diagramas de seta representado o campo no interior de uma guia de onda em distintos instantes de tempo.

Em ambas as situações, temos o fator complicador de as fontes serem caracterizadas por funções desconhecidas a priori $\left(\mathrm{B}_{\mathrm{U}}\right)$ e por levarem às equações de campos elétrico e magnético de tipo solenoidal (fluxo nulo e circulação não nula). O tempo de retardo assume importância grande, em especial, na análise das relações entre fluxo, circulação e campo (5). A determinação de pontos no espaço (3) é também crucial para a avaliação deste tempo de retardo. Entendemos que problemas deste tipo são muito difíceis por causa destes complicadores. Sintetizamos a classificação secundária de situações de $\Theta$ e $\Phi$ baseada nos seus parâmetros na Figura 9. 
Figura 9. Classificação secundária de situações das classes $\Theta e$ Ф baseada nos parâmetros

\begin{tabular}{|c|l|}
\hline$C_{S}$ & Parâmetros \\
\hline & $\begin{array}{l}\text { Portador individual de carga elétrica em movimento arbitrário, carga elétrica, posição distancia } \\
\text { ponto-fonte, velocidade, velocidade relativa, aceleração, momento magnético, campo elétrico, } \\
\text { campo magnético, curva amperiana, superfície gaussiana, aspectos geométricos (analógicas), } \\
\text { aspectos algébrico-analíticos (simbólicas), fluxo elétrico, fluxo magnético, circulação elétrica, } \\
\text { circulação magnética, campo elétrico, campo magnético. }\end{array}$ \\
\hline$\Theta_{\mathrm{B}}$ & $\begin{array}{l}\text { Todos parâmetros possíveis para } \Phi_{\mathrm{A}} \text { e } \Theta_{\mathrm{A}} \text { e distribuição discreta conhecida de portadores de } \\
\text { carga elétrica em movimento arbitrário substituindo o portador individual de carga elétrica em } \\
\text { movimento arbitrário }\end{array}$ \\
\hline$\Theta_{B}$ & $\begin{array}{l}\text { Todos os parâmetros possíveis para } \Phi_{B} \text { e } \Theta_{B} \text {, distribuições contínuas e conhecidas de portadores } \\
\text { de carga em movimento arbitrário, substituem as distribuições discretas e contínuas de } \\
\text { portadores de carga em movimento, mais carga elétrica, densidade de carga elétrica variável } \\
\text { no tempo, corrente elétrica, densidade de corrente elétrica variável no tempo, polarização, } \\
\text { magnetização, campo de polarização, campo de indução magnética }\end{array}$ \\
\hline$\Phi_{D}$ & $\begin{array}{l}\text { Todos os parâmetros possíveis para } \Phi_{C} \text { e } \Theta_{C} \text { e substituição de distribuições contínuas e } \\
\text { conhecidas de portadores de carga em movimento por distribuições contínuas e desconhecidas } \\
\text { de portadores de carga em movimento }\end{array}$ \\
$\Theta_{D}$
\end{tabular}

A classe de situações de cálculo do campo eletromagnético (T) requer o recrutamento de conceitos e operações de pensamento distintas das classes anteriores (Pantoja e Moreira, 2019). A quarta operação de pensamento geral é determinante para a distinção desta classe com relação às outras. Todos os conhecimentos derivados dos campos conceituais da álgebra, da geometria e da análise são relevantes para o cálculo do campo eletromagnético, portanto, não há preferência de um sobre o outro no domínio das situações. A resolução de situações de cálculo do campo eletromagnético requer como operações de pensamento gerais:

1. reconhecimento de fontes de campo eletromagnético ao longo do tempo;

2. reconhecimento de aspectos geométricos e analíticos da fonte ao longo do tempo;

3. determinação de distâncias entre as fontes e pontos do espaço onde se calcula o campo;

4. execução do cálculo do campo eletromagnético através de leis físicas;

5. interpretação do resultado obtido;

A execução do cálculo do campo eletromagnético usando leis físicas pode ser dividida em pelo menos oito operações de pensamento específicas. As duas primeiras são intituladas aplicação de leis adequadas para o cálculo do campo elétrico $\left(A_{\mathrm{T}}\right)$ e aplicação de leis adequadas para o cálculo do campo magnético $\left(B_{\mathrm{T}}\right)$, e servem à a necessidade da realização do cálculo operacional dos campos elétrico e magnético a partir de lei física adequada. As próximas duas são rotuladas cômputo do módulo do campo elétrico a diferentes distâncias $\left(C_{\mathrm{T}}\right)$ e cômputo do módulo do campo magnético a diferentes distâncias $\left(D_{\mathrm{T}}\right)$ e são justificadas pelo fato de precisarmos relacionar o módulo dos campos às distâncias entre ponto e fonte. Em seguida, temos as $h_{s}$ determinação da direção e do sentido do campo elétrico $\left(E_{\mathrm{T}}\right)$ e determinação da direção e do sentido do campo magnético 
$\left(F_{\mathrm{T}}\right)$ que se associam à determinação da direção dos campos elétrico e magnético, nem sempre à disposição por meios matemáticos. A necessidade de incorporar o princípio da superposição justifica as duas últimas operações de pensamento, a saber aplicação do princípio da superposição para cálculo de campos elétricos $\left(G_{\mathrm{T}}\right)$ e aplicação do princípio da superposição para cálculo de campos magnéticos $\left(H_{\mathrm{T}}\right)$.

A interpretação física do resultado obtido envolve oito operações de pensamento específicas, quais sejam, contraste entre campo elétrico e lei de Gauss $\left(I_{\mathrm{T}}\right)$, contraste entre campo elétrico e lei de Gauss magnética $\left(J_{\mathrm{T}}\right)$, contraste entre campo elétrico e lei de Faraday $\left(K_{\mathrm{T}}\right)$, contraste entre campo elétrico e lei de Ampère-Maxwell $\left(L_{\mathrm{T}}\right)$, contraste entre campo magnético e lei de Gauss $\left(M_{\mathrm{T}}\right)$, contraste entre campo magnético e lei de Gauss magnética $\left(N_{\mathrm{T}}\right)$, contraste entre campo magnético e lei de Faraday $\left(O_{\mathrm{T}}\right)$, contraste entre campo magnético e lei de Ampère-Maxwell $\left(P_{\mathrm{T}}\right)$. O campo magnético deve ser não monopolar e solenoidal, enquanto o campo elétrico pode ser tanto monopolar como rotacional. As fontes de campo magnético são correntes elétricas e campo elétricos variáveis no tempo, já o campo elétrico tem como fontes cargas elétricas e campos magnéticos variáveis no tempo. A interpretação física do resultado em termos das relações entre campo e equações de campo é imprescindível. A Figura 10 sistematiza as operações de pensamento primárias e secundárias relativas à classe de situações $\mathrm{T}$.

A seguir, indicamos as quatro classes secundárias de situações produzidas para esta classe primária com base nos parâmetros possíveis a ela. São elas, o cálculo do campo eletromagnético no espaço devido a:

- um portador pontual de carga elétrica em movimento arbitrário $\left(T_{\mathrm{A}}\right)$;

- um conjunto de portadores pontuais de cargas elétricas em movimento arbitrário $\left(\mathrm{T}_{\mathrm{B}}\right)$;

- um sistema de cargas e correntes elétricas distribuído continuamente, de forma conhecida a priori e variável no tempo $\left(\mathrm{T}_{\mathrm{C}}\right)$;

- um sistema de cargas e correntes elétricas distribuído continuamente, de forma desconhecida a priori e variável no tempo $\left(T_{\mathrm{D}}\right)$. 
Figura 10. Relação entre operações de pensamento primárias e secundárias para a classe primária de situações cálculo do campo eletromagnético (T)

\begin{tabular}{|c|c|c|}
\hline $\mathbf{h}_{\mathrm{p}}$ & $\mathbf{h}_{\mathrm{s}}$ & Descrição \\
\hline \multirow{2}{*}{1} & $A_{U}$ & Identificação de fontes de campo eletromagnético \\
\hline & $B_{U}$ & Caracterização de fontes de campo eletromagnético \\
\hline \multirow{4}{*}{2} & $A_{G}$ & Identificação de aspectos geométricos das fontes \\
\hline & $A_{A}$ & Identificação de aspectos algébrico-analíticos das fontes \\
\hline & $B_{G}$ & Caracterização de aspectos geométricos da fonte ao longo do tempo \\
\hline & $B_{A}$ & Caracterização de aspectos analítico-algébricos da fonte ao longo do tempo \\
\hline \multirow{3}{*}{3} & $A_{V}$ & Determinação de posições de pontos do espaço \\
\hline & $B_{V}$ & Determinação de posições de fontes ao longo do tempo \\
\hline & $C_{V}$ & Determinação de distâncias entre ponto e fontes ao longo do tempo \\
\hline \multirow{8}{*}{4} & $A_{T}$ & Aplicação de leis adequadas para o cálculo do campo elétrico \\
\hline & $B_{T}$ & Aplicação de leis adequadas para o cálculo do campo magnético \\
\hline & $C_{T}$ & Cômputo do módulo do campo elétrico a diferentes distâncias \\
\hline & $D_{T}$ & Cômputo do módulo do campo magnético a diferentes distâncias \\
\hline & $E_{T}$ & Determinação da direção e do sentido do campo elétrico \\
\hline & $F_{T}$ & Determinação da direção e do sentido do campo magnético \\
\hline & $G_{T}$ & Aplicação do princípio da superposição para cálculo de campos elétricos \\
\hline & $H_{T}$ & Aplicação do princípio da superposição para cálculo de campos magnéticos \\
\hline \multirow{8}{*}{5} & $I_{T}$ & Contraste entre o resultado obtido para o campo elétrico e a lei de Gauss \\
\hline & $J_{T}$ & Contraste entre o resultado obtido para o campo elétrico e a lei de Gauss magnética \\
\hline & $K_{T}$ & Contraste entre o resultado obtido para o campo elétrico e a lei de Faraday \\
\hline & $L_{T}$ & Contraste entre o resultado obtido para o campo elétrico e a lei de Ampère-Maxwell \\
\hline & $M_{T}$ & Contraste entre o resultado obtido para o campo magnético e a lei de Gauss \\
\hline & $N_{T}$ & Contraste entre o resultado obtido para o campo magnético e a lei de Gauss magnética \\
\hline & $O_{T}$ & Contraste entre o resultado obtido para o campo magnético e a lei de Faraday \\
\hline & $P_{T}$ & Contraste entre o resultado obtido para o campo magnético e a lei de Ampère-Maxwell \\
\hline
\end{tabular}

A classe primária de situações $\mathrm{T}$ envolve basicamente os mesmos objetos e variáveis que as classes de situações $\Phi$ e $\Theta$, mas as incógnitas variam radicalmente porque requerem a construção de uma expressão matemática envolvendo a descrição do campo eletromagnético. Por isto, haverá diferença nas operações de pensamento primárias e secundárias empregadas para determinar as incógnitas do problema. No caso do cálculo de campos magnéticos, há a união de operações de pensamento das classes primárias $\Phi$ e $\Theta$ relativas ao reconhecimento de aspectos geométricos, algébricos e analíticos das fontes ao longo do tempo, então todas estas variáveis respectivas a essas duas classes de situação estão empregadas nesta ação. Isto posto, iremos discutir os exemplos com vista a diferenciar cada uma das classes de situações secundárias das classes secundárias potencialmente similares $\left(\mathrm{T}_{j} \neq \Phi_{j} \neq \Theta_{j}\right)$ e a diferenciar uma classe secundária da outra $\left(\mathrm{T}_{i} \neq \mathrm{T}_{j}\right)$. 
Suponha um portador de carga pontual, F, movendo-se com velocidade $\vec{v}_{F}(t)=\left(v_{0}+a t\right) \hat{x}$. F está distante de $d$ de um ponto $P$ (ao longo do eixo $x$ ) em $t=$ 0 . Que funções descreveriam o campo elétrico e o campo magnético no ponto $P$ ?

Os objetos e variáveis são os mesmos das situações $\Theta_{A}$ e $\Phi_{A}$, mas dessa vez as incógnitas são duas funções de várias variáveis reais a valores vetoriais que descrevam os campos elétrico e magnético no espaço e no tempo. As $h_{G}$ de 1 a 4 , com exceção das $h_{s}$ $G_{\mathrm{T}}$ e $H_{\mathrm{T}}$, precisam ser conscientemente processadas para resolver problemas desse tipo. A $h_{G} 5$ é necessária para interpretação do resultado, mas pode parecer desnecessária caso o sujeito sustente uma visão instrumentalista do cálculo do campo eletromagnético.

Suponha dois portadores de cargas pontuais $F$ e $G$ movendo-se, respectivamente, com $\vec{v}_{F}(t)=\left(v_{0}+a t\right) \hat{x}$ e $\vec{v}_{G}(t)=\left(v_{0}+a t\right) \hat{y}$. Os portadores de carga elétrica estão inicialmente em $(0,0,0)$. Que função descreve o campo magnético resultante no espaço para qualquer instante de tempo?

Os objetos e variáveis são os mesmos das situações $\Theta_{B}$ e $\Phi_{B}$, mas a incógnita é uma função de várias variáveis reais a valores vetoriais descrevendo o campo magnético no espaço. Neste caso, a mudança dos objetos e variáveis ocasiona a necessidade de uso de $G_{\mathrm{T}}$ e $H_{\mathrm{T}}$ como $h_{S}$ para a $h_{G} 4$. A conceitualização se torna mais difícil por causa dessa consideração adicional.

Um solenoide longo tem diâmetro de $12,6 \mathrm{~cm}$. Quando a corrente $i$ percorre espiras, um campo magnético de $28,6 m T$ é gerado em seu interior. Diminuindo $i$, o campo descresce a uma taxa de $6,5 \mathrm{mT} / \mathrm{s}$. Calcule a intensidade do campo elétrico induzido a $2,20 \mathrm{~cm}$ a partir do eixo do solenoide.

Os objetos são um solenoide condutor de corrente e pontos do espaço. As variáveis são o diâmetro do solenoide, o campo magnético produzido por ele, a taxa de decréscimo do campo magnético e a distância ao ponto em questão. A incógnita é o valor do campo elétrico no ponto e ao longo do tempo. Este caso reforça aquele já discutido para as situações $\Sigma_{C}$, mas aplicado para o cálculo de um campo elétrico induzido por um campo magnético. Este exemplo ilustra a necessidade de escolha de operações de pensamento a serem realizadas, pois neste caso não são considerados os efeitos de variações temporais do campo elétrico, ou seja, corrente de deslocamento. Os parâmetros determinam os contextos das situações dentro do campo conceitual da indução eletromagnética. Na sequência, é trazido um exemplo da classe de situações $\mathrm{T}_{D}$.

Qual o campo eletromagnético resultante dentro de uma cavidade ressontante? Interprete fisicamente o resultado.

Este problema é parecido aos das classes $\Phi_{D}$ e $\Theta_{D}$, porém tem como objeto uma cavidade ressonante. As variáveis são semelhantes, inclusive. Contudo, a incógnita é uma função de várias variáveis reais a valores vetoriais representando o campo eletromagnético que deve ser calculada a partir da equação de onda. Os cálculos envolvem a forma diferencial das equações de Maxwell $\left(A_{\mathrm{T}}\right.$ e $\left.B_{\mathrm{T}}\right)$, que resultam em 
equações diferenciais de onda. A aplicação das condições de contorno e determinação do campo eletromagnético envolvem o resto das $h_{S}$ da $h_{G} 4$. Neste exemplo, solicitamos o emprego da $h_{G} 5$ ao agregarmos o comando "interprete fisicamente o resultado", pois o estudante deverá relacionar de forma coerente o campo eletromagnético com as leis que o sustentam.

A classificação secundária de situações da classe T, feita com base nos parâmetros, foi sintetizada na Figura 11.

Figura 11. Classificação secundária de situações da classe baseada nos parâmetros

\begin{tabular}{|c|l|}
\hline$C_{s}$ & Parâmetros \\
\hline$T_{A}$ & $\begin{array}{l}\text { Portador individual de carga elétrica em movimento arbitrário, carga elétrica, velocidade, } \\
\text { aceleração, posição do ponto, posição da fonte, distancia ponto-fonte, momento magnético, campo } \\
\text { elétrico, campo magnético, curva amperiana, superfície gaussiana, aspectos geométricos, aspectos } \\
\text { algébrico-analíticos, fluxo elétrico, fluxo magnético, circulação elétrica, circulação magnética, } \\
\text { campo elétrico, campo magnético, energia eletromagnética, momentum eletromagnético. }\end{array}$ \\
\hline$T_{B}$ & $\begin{array}{l}\text { Todos parâmetros possíveis para } \mathrm{T}_{A} \text {, distribuição discreta conhecida de portadores de carga } \\
\text { elétrica em movimento arbitrário substituindo o portador individual de carga elétrica em } \\
\text { movimento arbitrário, campo elétrico resultante e campo magnético resultante }\end{array}$ \\
\hline$T_{C}$ & $\begin{array}{l}\text { Todos os parâmetros possíveis para } \mathrm{T}_{B} \text {, distribuições contínuas e conhecidas de portadores de } \\
\text { carga em movimento arbitrário substituem as distribuições discretas de portadores de carga em } \\
\text { movimento arbitrário, mais densidade de carga elétrica variável no tempo, densidade de corrente } \\
\text { elétrica variável no tempo, carga elétrica, corrente elétrica, magnetização, polarização, campo de } \\
\text { deslocamento elétrico e campo de indução magnética. }\end{array}$ \\
\hline$T_{D}$ & $\begin{array}{l}\text { Todos os parâmetros possíveis para } \mathrm{T}_{C} \text {, substituição de distribuições contínuas e conhecidas de } \\
\text { portadores de carga em movimento por distribuições contínuas e desconhecidas de portadores } \\
\text { de carga em movimento }\end{array}$ \\
\hline
\end{tabular}

\section{Conclusões e implicações}

Neste trabalho, classificamos situações do campo conceitual da indução eletromagnética em quatro classes primárias de situações, são elas: descrição de interações eletromagnéticas $(\boldsymbol{\Sigma})$, representação analógica de campos eletromagnéticos $(\Phi)$, representação simbólica de campos eletromagnéticos $(\Theta)$ e cálculo do campo eletromagnético (T). Cada uma destas classes foi submetida a uma classificação secundária realizada com base em seus parâmetros. Esta classificação complementa as propostas de Pantoja e Moreira $(2019 ; 2020)$ para os campos conceituais da eletrostática e da magnetostática, no contexto da TE.

Esta abordagem ao conceito de indução eletromagnética evidencia as três facetas do eletromagnetismo, a saber, fonte, força e energia (Nousianen \& Koponen, 2017). Ontologicamente, o campo eletromagnético aparece desvinculado da força, pois agora possui uma velocidade de propagação e isto impõe um tempo de retardo, pois as interações eletromagnéticas não são instantâneas ${ }^{14}$. Com efeito, as três facetas

14 Embora muitos fenômenos de "correntes lentas" possam ser abordados de forma quase-estática e equivalham à perspectiva da ação à distância, o caso geral envolve a propagação dos campos eletromagnéticos no vácuo como ondas (Jackson, 1999). 
encontram-se relacionadas em estruturas conceituais epistemologicamente distintas nas quatro classes de situações. Por exemplo, as situações $\Sigma$ enfatizam trocas de energia (e momentum) por meio da ação da força exercida pelo campo, enquanto as $\mathbf{T}$ relacionam mais diretamente como determinadas fontes produzem certos campos (que carregam energia).

Destacamos a necessidade de descrição de contextos dentro do campo conceitual da indução eletromagnética, pois há uma diferenciação epistemológica em alguns fenômenos a serem abordados nele. Por exemplo, em situações de FEM de movimento existe o fenômeno de indução eletromagnética em regiões de campo magnético estacionário. Outro caso é o das correntes que variam lentamente e implicam campos eletromagnéticos quase-estáticos, o que nos permite ignorar a corrente de deslocamento na lei de Ampère-Maxwell (Jackson, 1999). Um terceiro tipo de situações é aquele no qual temos produção de ondas eletromagnéticas, de fato. Nessa esteira, é possível ainda pensar em contextos nos quais tratamos a corrente de deslocamento isolada dos campos magnéticos que indiretamente produz, por uma razão didática e não epistemológica. Cada um desses casos requer operações de pensamento distintas, mas somente o terceiro implica no uso de todas elas.

Os campos eletromagnéticos variam tanto com respeito à posição como com respeito ao tempo, no contexto da indução eletromagnética. Em geral, a complexidade das classes de situações cresce conforme se aumenta o número de variáveis e de objetos envolvidos, ou seja, as classes $\mathrm{D}$ costumam ser mais complicadas que a $\mathrm{B}$, que são mais exigentes que as A. Uma exceção é quando um campo elétrico dado apresenta simetria espacial, mas é variável com o tempo. Há casos nos quais é necessário calcular a FEM induzida em um circuito disposto em região de campo magnético ou elétrico dessa natureza como, por exemplo, entre as placas de um capacitor com corrente alternada $\left(\mathbf{T}_{\mathrm{C}}\right)$. Neste cenário, os cálculos são menos complicados do que os do campo eletromagnético de Jefimenko, das classes $\mathbf{T}_{\mathrm{A}}$, pois podem ser feitos a partir de equações integrais facilmente resolvíveis ${ }^{15}$. Por outro lado, o campo eletromagnético de uma carga pontual varia tanto na posição como no tempo e as densidades de carga e de corrente devem ser avaliadas no tempo retardado, que depende da posição. Fora este caso particular, as expressões gerais para os campos eletromagnéticos devidos a sistemas contínuos de cargas elétricas e correntes elétricas são bem mais complicados que os da classe $\mathrm{T}_{\mathrm{A}}$.

A educação escolar pode ser mais profundamente pesquisada se os conhecimentos são organizados em campos conceituais. Nesta perspectiva, ensinar envolve prover situações que possam ser entendidas pelos estudantes como problemas e lhes permitam atribuir sentido aos conceitos. Conforme discutido, a complexidade das situações se relaciona à das operações de pensamento necessárias para resolvê-las que, por sua vez, associam-se à quantidade de parâmetros nas situações. Esta classificação provê condições para o professor construir suas próprias situações de modo que elas estejam dentro da zona de desenvolvimento proximal dos estudantes (Vergnaud, 2007). Ao pesquisador, torna-se possível analisar que formas de situações são mais profícuas para

15 Lei de Ampère-Maxwell ou lei de Faraday, a depender do caso. 
facilitar processos de aprendizagem. Desta forma, destacamos a construção de um campo conceitual como primeira contribuição para a pesquisa em Ensino (Vergnaud, 1982; 2009; 2013).

A partir da construção supracitada, é possível investigar quais conhecimentos os estudantes possuem, sejam eles implícitos ou explícitos, qual o significado de diferentes representações simbólicas que os estudantes apresentam para determinado conceito, e como ocorre a interação entre esquemas e situações (Vergnaud, 2013). Tudo isto permite conhecer como estruturas de conhecimento operatório são mobilizadas em situação. Possibilitar a sistematização do estudo do conhecimento operatório é outra vertente deste trabalho que pode ser apontada como aporte para a pesquisa em Ensino de Ciências.

Os parâmetros da situação influenciam na conceitualização e isto é verificado em outros campos conceituais (Vergnaud, 1982; Pantoja \& Moreira, 2019; Scaife \& Heckler, 2010). Isto pode ser destacado com respeito às classes primárias de representação analógica $(\Phi)$ e de representação simbólica $(\Theta)$ do campo eletromagnético. Duas situações podem ter os mesmos objetos e variáveis, mas é possível dispararem diferentes operações de pensamento por causa das suas incógnitas. A classificação secundária se torna essencial, pois leva em conta a dependência da conceitualização com os parâmetros da situação. Por exemplo, as situações dos tipos $\Sigma_{\mathrm{A}}$ e $\Sigma_{\mathrm{B}}$ diferem no número de teoremasem-ação e conceitos-em-ação recrutados para resolvê-las.

A partir desta proposta é possível investigar a influência da variação de parâmetros em uma classe secundária na conceitualização para estudantes de vários níveis. Por exemplo, existem evidências empíricas de que o simples fato de explicitar a fonte de campo magnético já dificulta bastante a situação para os estudantes (Scaife \& Heckler, 2010). Portanto, possibilitar a classificação terciária de situações é a terceira contribuição que enumeramos. Nesta esteira, está a descrição mais aprofundada e simultânea da cognição predicativa e operatória, que só se torna possível quando analisamos como ocorre a adaptação de esquemas, e de outros modos de conceitualização, às situações (Pantoja \& Moreira, 2019; 2020). Tal ponto está dentro de nossas perspectivas futuras de pesquisa.

Por último, podemos perguntar-nos: "por que alunos costumam mobilizar conhecimentos diferentes em situações correlatas?” Muitas vezes, em especial quando os estudantes não possuem esquemas no seu repertório cognitivo, e a conceitualização se processa de forma não-sistemática, os estudantes costumam esgotar, de forma criativa, seus recursos cognitivos para o domínio da situação, o que é comumente conhecido como oportunismo da conceitualização (Fanaro, Otero \& Moreira, 2011). Neste processo, a conceitualização fica cada vez mais dependente dos parâmetros das situações, assim, nossa classificação permite caracterizar os parâmetros envolvidos na tarefa, o que possibilita estudar este fenômeno de forma mais sistemática. Aparentemente, modos oportunistas de conceitualização podem ser encontrados em uma reinterpretação do caso evidenciado por Guisasola et al. (2011), pois em situações correlatas, os estudantes parecem não ter um esquema formado para abordar aspectos microscópicos, mas 
parecem possui-los para abordar aspectos macroscópicos. Portanto, nos primeiros tipos de situação, precisam encontrar uma forma de resolver as situações com os recursos cognitivos disponíveis enquanto no segundo, já parecem dispor de um esquema prévio.

\section{Referências}

Albe, V., Venturini, P., \& Lascours, J. (2001). Electromagnetic Concepts in Mathematical Representation of Physics. Journal of Science Education and Technology, 10(2), 197-203.

Bagno, E., \& Eylon, B. S. (2000). From problem solving to a knowledge structure: An example from the domain of electromagnetism. American Journal of Physics, 65(8), 726736.

Bunge, M. (2011). Caçando a realidade. Perspectiva.

Galili, I., Kaplan, D., \& Lehavy, Y. (2006). Teaching Faraday's law of electromagnetic induction in an introductory physics course. American Journal of Physics, 74(4), 337343.

Griffiths, D. J. (1999) Introduction to Electrodynamics. Prentice Hall.

Guisasola, J. Almudí, J. M., \& Zuza, K. (2011). University Students' Understanding of Electromagnetic Induction. International Journal of Science Education. 35(16), 26922717.

Jackson, J. D. (1999) Classical Electrodynamics (3th ed). John Wiley and Sons.

Jelicic, K., Planinic, M., \& Plasinic, G. (2017) Analyzing high school students' reasoning about electromagnetic induction. Physical Review Physics Education Research, 13(1), 010112.

Kuhn, T. (1997) A estrutura das revoluções científicas. Editora Perspectiva.

Loftus, M. (1996). Students' ideas about electromagnetism. SSR, 77, 280.

Mauk, H. V. \& Hingley, D. (2005). Student understanding of induced current: Using tutorials in introductory physics to teach electricity and magnetism. American Journal of Physics, 73(12), 1164-1171.

Nousianen, M., \& Koponen, I. (2017) Pre-service physics teachers' content knowledge of electric and magnetic field concepts: Conceptual facets and their balance. European Journal of Science and Mathematics Education. 5(1), 74-20.

Pantoja, G. C., \& Moreira, M. A. M. (2019). Classificação de problemas em eletrostática: uma análise epistemológica rumo à construção de um campo conceitual para o conceito de campo eletrostático. Latin American Journal of Physics Education, 13(4), 4304.

Pantoja, G. C., \& Moreira, M. A. M. (2020). Campos Conceituais e Campo Magnético: classificação epistemológica de tarefas em magnetostática. Não publicado. 
Pantoja, G. C., Moreira, M. A. M., \& Herscovitz, V. E. (2011). Uma revisão da literatura sobre a pesquisa em Ensino de Mecânica Quântica no período de 1999 a 2009. Revista Brasileira de Ensino de Ciência e Tecnologia, 4, 1-34.

Resnick, R., Halliday, D., \& Krane, K. (2006) Física: volume 3 (quinta edição). LTC.

Saarelainen, M., Laaksonen, A., \& Hirvonen, P. E. (2007). Students' initial knowledge of electric and magnetic fields - more profound explanations and reasoning models for undesired conceptions. European Journal of Physics, 28, 51-60.

Scaife, T., \& Heckler, A. (2010) Student understanding of the direction of the magnetic force on a charged particle. American Journal of Physics. 78(8), 869-876.

Thong, W., \& Gunstone, R. (2008). Some Students Conceptions of Electromagnetic Induction. Research in Science Education, 38, 31-44.

Venturini, P., \& Albe, V. (2002). Interpretation des similitudes et differences dans la maîtrise conceptualle d'etudiants en electromagnetism a partir de leur(s) rapport(s) au(x) savoir(s). ASTER, 25, 165-188.

Vergnaud, G. (1982) A classification of cognitive tasks and operations of thought involved in addition and subtraction problems. In T. Carpenter, J. Moser, \& T. Romberg, (Eds.) Addition and subtraction. A cognitive perspective. Lawrence Erlbaum, 39-59.

Vergnaud, G. (2009) The Theory of Conceptual Fields. Human Development, 52(2), 8394.

Vergnaud, G. (2013) Pourquoi la théorie des champs conceptuels? Infancia y Aprendizaje, $36(2), 131-161$.

Whittaker, E. (1910) A history of the theories of aether and electricity: From the age of Descartes to the close of the nineteenth century. Kessinger Publishing.

Zuza, K., Almudí, J., \& Guisasola, J. (2012a) Revisión de la investigación acerca de las ideas de los estudiantes sobre la interpretación de los fenómenos de inducción electromagnética. Enseñanza de las ciencias, 30(2), 175-196.

Zuza, K., Almudí, J., Leniz, A., \& Guisasola, J. (2014) Adressing students' difficulties with Faraday's law: a guided problem-solving approach. Physical Review Special Topics Physics Education Research, 10(1), 1-16.

Zuza, K., Guisasola, J., Michelini, M., \& Santi, L. (2012b). Rethinking Faraday's law for teaching motional electromotive force. European Journal of Physics, 33, 397-406. 


\section{Glauco Cohen Pantoja}

Universidade Federal do Oeste do Pará

Instituto de Ciências da Educação

Santarém, Pará, Brasil

glaucopantoja@hotmail.com

\section{Editor Responsável}

Alessandro Damásio Trani Gomes

Manifestação de Atenção às Boas Práticas Científicas e de Isenção de Interesse

Os autores declaram ter cuidado de aspectos éticos ao longo do desenvolvimento da pesquisa e não ter qualquer interesse concorrente ou relações pessoais que possam ter influenciado o trabalho relatado no texto. 